\title{
Hipoeliticidade Global e Resolubilidade para Algumas Classes de Operadores Diferenciais
}

Edna Maura Zuffi

\author{
Orientação \\ Prof. Dr. Adalberto Panobianco Bergamasco
}

Dissertação apresentada ao Instituto de Ciências Matemáticas de São Carlos USP, como parte dos requisitos para obtenção do título de Mestre em Matemática.

USP - São Carlos

outubro de 1991 
Aos meus pais e à Clélia 
"... amanhã será outro dia

para ele viajas

vamos para ele.

Venceste o desgosto, calcaste o individuo,

já teu passo avança

em terra diversa.

Teu passo: outros passos

ao lado do teu.

O pisar de botas,

outros nem calçados,

mas todos pisando,..."

(Uma hora e mais outra)

Carlos Drummond de Andrade

Há homens que lutam um dia e são bons;

há homens que lutam um ano e são melhores;

há outros que lutam muitos anos, e são muito bons;

porém, há os que lutam por toda a vida

$e$ estes são os imprescindíveis.

Bertold Brécht 
Agradeço a meus pais e ao Édson, pelo carinho infindável e pela confiança sempre presentes; à Clélia, pela grande oportunidade que me concedeu; ao Dr. Fernado Luis Benini Reis, pela dedicação com que cuidou do restabelecimento de minha saúde, sem o qual não teria concluido este trabalho; ao Prof. Adalberto, pela excelente orientação e pela amizade, imprescindíveis nos momentos difíceis; aos professores do Instituto -em particular ao Hermano, ao Biasi e ao Wagner - pelo incentivo e pelas discussões; aos colegas, pela atençấo e carinho, e a Deus, pela oportunidade de ter conhecido tantas pessoas maravilhosas.

Este trabalho contou com apoio parcial do $\mathrm{CNPq}$ 


\begin{abstract}
In this work we use characterizations of periodic distributions and smooth periodic functions by means of their Fourier series to study global hypoellipticity and global solvability on the $n$-torus $(n \geq 2)$ for a class of partial differential operators. Some of these have constant coefficients and others, variable and smooth ones.
\end{abstract}




\section{Resumo}

Neste trabalho, utilizamos as caracterizações de distribuições periódicas e de funções periódicas suaves por meio de suas séries de Fourier, para estudarmos a hipoeliticidade global e a resolubilidade global no $n$-toro $(n \geq 2)$ para uma classe de operadores diferenciais parciais, onde alguns destes têm coeficientes constantes e outros, coeficientes variáveis e suaves. 


\section{Conteúdo}

1 Pré-Requisitos 1

1.1 Espaços de Funções Testes e de Distribuições Periódicas . . . . . 2

1.2 Operadores elíticos, hipoelíticos e globalmente hipoelíticos . . . 5

1.3 Exemplos de operadores elíticos, $(\mathrm{H})$ e $(\mathrm{GH}) \ldots \ldots \ldots$

1.4 Os espaços de Sobolev . . . . . . . . . . . . . . . 6

1.5 Alguns resultados de Análise . . . . . . . . . . . 8

1.5.1 Teorema da aplicação aberta para espaços de Fréchet . . . 8

1.5.2 Operadores lineares contínuos e semi-normas . . . . . . 9

1.5.3 Existência e regularidade de solução para uma E.D.O. . . . 9

2 Hipoeliticidade e Resolubilidade Globais para Alguns Operadores a Coeficientes Constantes 11

2.1 Hipoeliticidade Global e Resolubilidade em $T^{n} \ldots \ldots$. . . . . 12

2.2 Resolubilidade em $C_{S}^{\infty}\left(T^{n}\right) \ldots \ldots \ldots \ldots \ldots \ldots$

2.3 Exemplos de operadores $(\mathrm{GH})$ e operadores (GR) . . . . . . 21

2.3.1 Exemplo 1................... 26

2.3.2 Exemplo 2................... 26

2.3.3 Exemplo $3 \ldots \ldots \ldots \ldots \ldots$ 
2.3 .4 Exemplo $4 \ldots \ldots \ldots \ldots \ldots \ldots \ldots \ldots \ldots$

2.3.5 Exemplo $5 \ldots \ldots \ldots \ldots \ldots \ldots \ldots \ldots$

2.3 .6 Exemplo $6 \ldots \ldots \ldots \ldots \ldots \ldots \ldots \ldots$

3 Hipoeliticidade Global para uma Classe de Operadores em $T^{n}$ com Coeficientes Variáveis

3.1 Hipoeliticidade Global para um Operador da forma: $P=p\left(D_{1}^{2}\right)+$ $e^{i x_{1}}+a e^{-i x_{1}} \ldots \ldots \ldots \ldots \ldots \ldots \ldots \ldots \ldots \ldots \ldots \ldots$

3.1 .1 Exemplo $1 \ldots \ldots \ldots \ldots \ldots \ldots \ldots \ldots$

3.1 .2 Exemplo $2 \ldots \ldots \ldots \ldots \ldots \ldots \ldots \ldots \ldots$

3.1.3 Exemplo $3 \ldots \ldots \ldots \ldots \ldots \ldots \ldots \ldots$

3.2 Alguns Resultados sobre uma Equação de Diferenças . . . . . 35

3.3 Demonstração do teorema central . . . . . . . . . . . 41

3.4 Observaşões relevantes sobre o teorema central . . . . . . . 52

3.4 .1 A unicidade da solução . . . . . . . . . . . . 52

3.4.2 O operador $P=D_{1}^{2}+2 r i \operatorname{sen} x_{1}, \operatorname{com} r>1 \ldots \ldots 2$

3.4.3 Por que utilizar grau $p a r-$ ou $p\left(D_{1}^{2}\right) \quad \ldots \ldots \ldots 56$

3.5 A hipoeliticidade global para o operador $Q=q\left(D^{\prime}\right)+2 \cos x_{1} \ldots \quad 58$

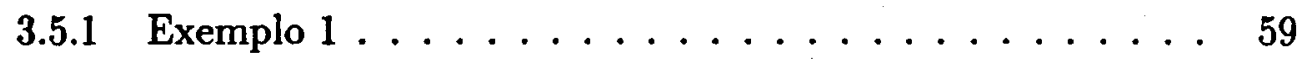

3.5 .2 Exemplo $2 \ldots \ldots \ldots \ldots \ldots \ldots \ldots \ldots \ldots$

3.5.3 Demonstração do teorema $\ldots \ldots \ldots \ldots \ldots$. . . . 60

3.5 .4 Outra classe de operadores $\ldots \ldots \ldots \ldots \ldots \ldots$

$\begin{array}{ll}\text { A } & 65\end{array}$ 


\section{Introdução}

Neste trabalho, nosso principal objetivo é estudar a hipoeliticidade global para algumas classes de operadores diferenciais parciais lineares, agindo em funções definidas no toro $n$-dimensional $T^{n}, n \geq 2$. Tal propriedade trata da regularidade de soluções sempre que o segundo membro de uma E.D.P. é regular (função infinitamente diferenciável). A definição de um operador globalmente hipoelítico (GH) na qual estamos particularmente interessados é a seguinte:

$$
P \text { é (GH) se, e somente se, }
$$

as condições: $u \in \mathcal{D}^{\prime}\left(T^{n}\right)$ e $P u=f \in C^{\infty}\left(T^{n}\right)$

implicam $u \in C^{\infty}\left(T^{n}\right)$.

Outro objetivo importante ao qual nos propomos é verificar algumas hipóteses sob as quais estes operadores são globalmente resolúveis (GR), ou seja, sob que condições o operador: $P: X \rightarrow X$ é sobrejetor, quando $X \subset C^{\infty}\left(T^{n}\right)$ é um subespaço.

Para isto, no primeiro capítulo introduzimos várias definições e resultados importantes a serem utilizados no estudo das propriedades descritas anteriormente. Enunciamos os principais teoremas que tratam das caracterizações de funções periódicas suaves (infinitamente diferenciáveis) e de distribuições periódicas, por meio de suas séries de Fourier (seção 1.1). Destacamos, ainda, alguns resultados de Análise que serão utilizados ao longo deste trabalho (seção 1.5) e um breve estudo dos espaços de Sobolev para funções periódicas (seção 1.4).

No segundo capítulo, estudamos a hipoeliticidade global (HG) para operadores a coeficientes constantes da forma:

$$
\begin{gathered}
P=\sum_{|\alpha| \leq m} c_{\alpha} D_{1}^{\alpha_{1}} \ldots D_{n}^{\alpha_{n}}, \text { onde } c_{\alpha} \in \mathbf{C}, D_{j}=\frac{1}{i} \frac{\partial}{\partial x_{j}}, \\
\alpha \in \mathbf{Z}_{+}^{n},|\alpha|=\alpha_{1}+\cdots+\alpha_{n}
\end{gathered}
$$


e destacamos um resultado de [G-W] (seção 2.1) que trata da resolubilidade de $P$ em $C^{\infty}\left(T^{n}\right)$ - a menos de um espaço de dimensão finita - e sua relação com a hipoeliticidade global:

Teorema 0.1 Se $P$ é (GH), então $P$ é Fredholm de indice zero, i.e., $\operatorname{dim} \operatorname{Ker} P=$ codim $\operatorname{Im} P$.

Outro operador, tratado em [Mos], e analisado neste capitulo é:

$$
\begin{gathered}
L=\sum_{j=1}^{n} \tilde{D}_{j}^{2}: C_{0}^{\infty}\left(T^{n+1}\right) \rightarrow C_{0}^{\infty}\left(T^{n+1}\right), \text { onde } \\
\tilde{D}_{j}=\partial x_{j}-\alpha_{j} \partial x_{n+1}, \alpha_{j} \in R, j=1,2, \ldots \mathrm{e} \\
C_{0}^{\infty}\left(T^{n+1}\right)=\left\{f \in C^{\infty}\left(T^{n+1}\right): \hat{f}(0)=(2 \pi)^{-(n+1)} \int_{T^{n+1}} f(x) d x=0\right\} .
\end{gathered}
$$

Com este tipo de operadores, traçamos um paralelo entre a (HG) e a (RG) (seção 2.2). Finalmente, analisamos vários exemplos nesta classe (seção 2.3) e sua relação com os números de Liouville.

No terceiro capítulo (seções 3.1, 3.2, 3.3 e 3.4), abordamos a hipoeliticidade global -tratada em [Yos] - para outra classe de operadores, agora com alguns coeficientes variáveis em $T^{n}$, do tipo:

$$
P=p\left(D_{1}^{2}\right)+e^{i x_{1}}+a e^{-i x_{1}}, \text { onde } a= \pm 1 .
$$

A demonstração do teorema central fornece uma maneira interessante de provarmos outros resultados (seção 3.5) acerca da hipoeliticidade global para operadores da forma:

$$
Q=q\left(D^{\prime}\right)+2 \cos x_{1}, \text { onde } q\left(D^{\prime}\right)=\sum_{\substack{\alpha \in Z_{n-1} \\|\alpha| \leq m}} c_{\alpha} D_{2}^{\alpha_{2}} \ldots D_{n}^{\alpha_{n}}, c_{\alpha} \in \mathbf{C},
$$

onde está envolvida a resolução de uma equação de diferenças do tipo:

$$
v_{j+2}=a_{j} v_{j+1}+v_{j}-f_{j}, j=0, \pm 1, \pm 2, \ldots, a_{j}, f_{j} \in \mathbf{C} .
$$

Finalizando, citamos outro resultado de [Yos] sobre a hipoeliticidade global para os operadores:

$$
\begin{gathered}
P=\sum_{|\alpha| \leq m} a_{\alpha} D^{\alpha}+\sum_{|\beta| \leq m-s} b_{\beta}(x) D^{\beta}, \text { onde } m \geq s \geq 1, a_{\alpha} \in \mathrm{C}, \\
b_{\beta} \in C^{\infty}\left(T^{n}\right) \text { e } \hat{b}_{\beta}(0)=(2 \pi)^{-n} \int_{T^{n}} b_{\beta}(x) d x=0, \forall \beta,|\beta| \leq m-s,
\end{gathered}
$$

os quais serão objetos de estudos futuros. 


\section{Capítulo 1}

\section{Pré-Requisitos}

O objetivo deste capítulo é apresentar a notação e destacar as definições importantes a serem utilizadas nos capítulos 2 e 3 , bem como os resultados que serviram como pré-requisitos para o desenvolvimento da teoria neste trabalho. $\mathrm{Na}$ seção (1.1) apresentamos os espaços de funções testes e de distribuições periódicas, e também sua relação com os espaços de sequências rapidamente decrescentes e de sequências lentamente crescentes. Na seção (1.2) introduzimos os conceitos de operadores elíticos, hipoelíticos e globalmente hipoelíticos, destacando alguns exemplos relevantes. Na seção (1:3) fazemos uma rápida abordagem sobre os espaços de Sobolev e sua relação com os espaços da seção (1.1). Finalizando este capítulo, destacamos dois importantes resultados de Análise a serem utilizados: o Teorema da Aplicação Aberta para Espaços de Fréchet e um Teorema de Existência de Solução para uma E.D.O. 


\subsection{Espaços de Funções Testes e de Distribuições Periódicas}

Definição $1.1 C^{\infty}\left(\mathbf{R}^{n}\right)$ é o espaço das funções infinitamente diferenciáveis, definidas em $\mathbf{R}^{n}$, a valores complexos.

Definição $1.2 C_{2 \pi}^{\infty}\left(\mathbf{R}^{n}\right)$ é o subespaço de $C^{\infty}\left(\mathbf{R}^{n}\right)$ formado pelas funções $2 \pi$ periódicas em cada variável.

Definição $1.3 C^{\infty}\left(T^{n}\right)$ é o espaço das funções infinitamente diferenciáveis definidas no toro $T^{n}$, a valores complexos, onde:

$$
T^{n} \cong \mathbf{R}^{n} / 2 \pi \mathbf{Z}^{n} ; n \geq 1
$$

Tem-se:

- $C^{\infty}\left(T^{n}\right) \cong C_{2 \pi}^{\infty}\left(\mathbf{R}^{n}\right)$.

- As seminormas $p_{k}(f)=\sup \left\{\left|\partial^{\alpha} f(x)\right| ; x \in T^{n},|\alpha| \leq k\right\}, k=1,2, \ldots$, e $p_{0}(f)=\sup \left\{|f(x)|, x \in T^{n}\right\}$ definem em $C^{\infty}\left(T^{n}\right)$ uma topologia localmente convexa, metrizável e completa.

Com isto, vemos que $C^{\infty}\left(T^{n}\right)$ é um espaço de Fréchet. Uma métrica que pode ser definida através das semi-normas $p_{k}, k=0,1, \ldots$ é:

$$
d(f, g)=\sum_{k=0}^{\infty} \frac{1}{2^{k}} \frac{p_{k}(f-g)}{1+p_{k}(f-g)}
$$

e temos, então, a topologia que fornece a convergência uniforme da função e de todas as suas derivadas.

Definição 1.4 $\mathcal{D}^{\prime}\left(T^{n}\right)$ é o espaço de todas as distribuiçôes no toro $T^{n}$, i.e., o espaço dos funcionais lineares contínuos $u: C^{\infty}\left(T^{n}\right) \rightarrow \mathrm{C}$. 
Definição 1.5 Se $f \in C^{\infty}\left(T^{n}\right)$ e $k=\left(k_{1}, \ldots, k_{n}\right) \in \mathbf{Z}^{n}$, definimos o $k$-ésimo coeficiente de Fourier de $f$ por:

$$
\begin{gathered}
\hat{f}(k)=\frac{1}{(2 \pi)^{n}} \int_{T^{n}} e^{-i k . x} f(x) d x=\left\langle f, e_{-k}>, \text { onde } e_{-k}(x)=e^{-i k . x}, i=\sqrt{-1}\right. \\
x=\left(x_{1}, \ldots, x_{n}\right) \in T^{n}, k . x=k_{1} x_{1}+\cdots+k_{n} x_{n} .
\end{gathered}
$$

A série de Fourier de $f$ é definida por: $\sum_{k \in Z^{n}} \hat{f}(k) e^{i k . x}$.

Teorema 1.1 Se $f \in C^{\infty}\left(T^{n}\right)$, então $f$ é igual à sua série de Fourier, i.e.,

$$
f(x)=\sum_{k \in Z^{n}} \hat{f}(k) e^{i k . x}
$$

com convergência na topologia de $C^{\infty}\left(T^{n}\right)$.

É possivel caracterizar uma função de $C^{\infty}\left(T^{n}\right)$ através de seus coeficientes de Fourier:

Teorema 1.2 Uma sequência $\left(a_{k}\right)_{k \in Z^{n}} \in \mathbf{C}$ é a sequência de coeficientes de Fourier de uma função $f \in C^{\infty}\left(T^{n}\right) \Leftrightarrow$ para cada $M \in \mathrm{N}$, existe $C>0$ tal que:

$$
\left|a_{k}\right| \leq C(1+|k|)^{-M}, k \in \mathbf{Z}^{n}, \text { onde }|k|=\sqrt{k_{1}^{2}+\cdots+k_{n}^{2}}
$$

Ou, equivalentemente:

$\alpha=\left(a_{k}\right)_{k \in Z^{n}}$ é a sequência de coeficientes de Fourier de uma função $f \in C^{\infty}\left(T^{n}\right) \Leftrightarrow$ para cada $M \in \mathrm{N} \cup\{0\}$

$$
\sum_{k \in \mathbf{Z}^{n}}\left|a_{k}\right||k|^{M}<\infty \text { ou } \sup \left\{\left|a_{k} \| k\right|^{M} ; k \in \mathbf{Z}^{n}\right\}<\infty
$$


Definição 1.6 Estas sequências são ditas rapidamente decrescentes e seu espaço será denotado por $S\left(\mathbf{Z}^{n}\right)$. Definimos em $S\left(\mathbf{Z}^{n}\right)$ as semi-normas:

$$
\|\alpha\|_{\infty, M}=\sup \left\{\left|a_{k}\right||k|^{M}, k \in \mathbf{Z}^{n}\right\}, M \in \mathbf{N}
$$

$\boldsymbol{e}$

$$
\|\alpha\|_{\infty, 0}=\sup \left\{\left|a_{k}\right|, k \in \mathbf{Z}^{n}\right\}
$$

e a métrica:

$$
\tilde{d}(\alpha, \beta)=\sum_{M=0}^{\infty} \frac{1}{2^{M}} \frac{\|\alpha-\beta\|_{\infty, M}}{1+\|\alpha-\beta\|_{\infty, M}}, \forall \alpha, \beta \in S\left(\mathbf{Z}^{n}\right) .
$$

Teorema 1.3 $O$ operador $\wedge: C^{\infty}\left(T^{n}\right) \rightarrow S\left(\mathbf{Z}^{n}\right)$, que a cada função $f \in C^{\infty}\left(T^{n}\right)$ associa a sequência dos seus coeficientes de Fourier: $(\hat{f}(k))_{k \in \mathbf{Z}^{n}}, e ́$ um isomorfismo e também um homeomorfismo, i.e., é uma bijeção linear contínua com inversa contínua (em relação às métricas de e d).

Definição 1.7 Se $u \in D^{\prime}\left(T^{n}\right)$, definimos o k-ésimo coeficiente de Fourier de u por:

$$
\hat{u}(k)=\frac{1}{(2 \pi)^{n}}\left\langle u, e_{-k}>\text {, onde } e_{k}=e^{i k . x} .\right.
$$

Teorema $1.4 u \in \mathcal{D}^{\prime}\left(T^{n}\right)$ é igual à sua série de Fourier ,i.e.,

$$
u=\sum_{k \in \mathbb{Z}^{n}} \hat{u}(k) e_{k},
$$

com convergência na topologia fraca de $\mathcal{D}^{\prime}\left(T^{n}\right)$, ou seja, para toda $\varphi \in C^{\infty}\left(T^{n}\right)$ tem-se:

$$
\left\langle u, \varphi>=(2 \pi)^{n} \sum_{k \in Z^{n}} \hat{u}(k) \cdot \hat{\varphi}(-k) .\right.
$$

Teorema 1.5 Uma sequência numérica $\alpha=\left(a_{k}\right)_{k \in Z^{n}} \in \mathbf{C}$ é a sequência dos coeficientes de Fourier de uma distribuição $u \in \mathcal{D}^{\prime}\left(T^{n}\right) \Leftrightarrow$ existem $C>0$ e $M \in \mathbf{N}$ tais que:

$$
\left|a_{k}\right| \leq C(1+|k|)^{M}, \forall k \in \mathbf{Z}^{n} .
$$

Estas são ditas sequências de crescimento lento e seu espaço será denotado por $S^{\prime}\left(\mathbf{Z}^{n}\right)$.

Teorema 1.6 Se $\alpha=\left(a_{k}\right)_{k \in Z^{n}}$ é uma sequência complexa de crescimento lento, então existe uma única $u \in \mathcal{D}^{\prime}\left(T^{n}\right)$ tal que $\hat{u}(k)=a_{k}, \forall k \in \mathbf{Z}^{n}$. Reciprocamente, se $u \in \mathcal{D}^{\prime}\left(T^{n}\right)$, então $\hat{u}=(\hat{u}(k))_{k \in Z^{n}}$ é de crescimento lento. ${ }^{1}$

\footnotetext{
${ }^{1}$ Referências: [Berg]; [Zan]; [Ior].
} 


\subsection{Operadores elíticos, hipoelíticos e global- mente hipoelíticos}

Seja $P(x, D)=\sum_{|\alpha| \leq m, \alpha \in \mathbf{Z}^{n}} a_{\alpha}(x) D^{\alpha}$ um operador diferencial em $X=T^{n}$ ou $\mathbf{R}^{n}$, onde $D^{\alpha}=D_{1}^{\alpha_{1}} \ldots D_{n}^{\alpha_{n}}$ e $D_{j}=\left(\frac{1}{i} \frac{\partial}{\partial x_{j}}\right)$.

Definição 1.8 $P$ é elítico em $x_{0} \in X$ se $P_{m}\left(x_{0}, \xi\right)=\sum_{|\alpha|=m} a_{\alpha}\left(x_{0}\right) \xi^{\alpha} \neq 0$ para todo $\xi \in \mathbf{R}^{n} \backslash\{0\}$.

Quando $P(x, D)$ é elítico em todos os pontos de $X$, dizemos que $P$ é elítico em $X$.

Definição $1.9 P$ é hipoelítico, $(H)$, em $X \Leftrightarrow \forall Y \subset X$ aberto, as condições: $u \in D^{\prime}(X)$ e $P u \in C^{\infty}(Y)$ implicam $u \in C^{\infty}(Y)$.

Definição 1.10 $P$ é globalmente hipoelítico, $(G H)$, em $X \Leftrightarrow$ as condições $u \in \mathcal{D}(X)$ e $P u \in C^{\infty}(X)$ implicam $u \in C^{\infty}(X)$.

Observemos que $\mathrm{P}$ é (H) em $\mathrm{X} \Rightarrow \mathrm{P}$ é (GH) em $\mathrm{X}$. Mas a recíproca não é verdadeira, como veremos em exemplos posteriores.

\subsection{Exemplos de operadores elíticos, (H) e (GH)}

Um exemplo clássico de operador elítico em $\mathbf{R}^{n}$ é o Laplaciano:

$$
\Delta=\sum_{j=1}^{n}\left(\frac{\partial}{\partial x_{j}}\right)^{2}=-\sum_{j=1}^{n} D_{j}^{2}
$$

o qual sabemos que é também (H) em $\mathbf{R}^{n}$ e, portanto, (GH) em $\mathbf{R}^{n}$ e $T^{n}$.

Outro exemplo de operador elítico, (H) e, portanto (GH) em $\mathbf{R}^{2}$ e $T^{2}$ é:

$$
\left.L=\frac{1}{2}\left(\frac{\partial}{\partial x}+i \frac{\partial}{\partial y}\right)=\frac{\partial}{\partial \bar{z}} \text { (operador de Cauchy }- \text { Riemann }\right)
$$

Já o operador de calor definido em $\mathbf{R}^{n}$ por:

$$
P=\frac{\partial}{\partial t}-\Delta
$$


não é elítico, mas pode-se mostrar que é (H) e, portanto, (GH) em $\mathbf{R}^{n+1}$ e $T^{n+1}$.

O operador das ondas em $\mathbf{R}^{n+1}$, definido por:

$$
\frac{\partial}{\partial t}^{2}-c^{2} \Delta
$$

também não é elítico; pode-se mostrar que não é $(H)$ em $\mathbf{R}^{n+1}$ e também não é (GH) em $\mathbf{R}^{n+1}$, mas pode ser $(\mathrm{GH})$ em $T^{n+1}$, dependendo do valor de $\underline{\mathbf{c}}$. 2 e 3.

Exemṕlos de operadores (GH) que não são (H) serão abordados nos capítulos

Um exemplo de operador que não é (GH) em $T^{2}$ é $: D_{1}-\gamma\left(x_{1}\right)$, com $\gamma$ satisfazendo: $\int_{0}^{2 \pi} \gamma(s) d s=0$, o qual será abordado no capítulo 3 .

Outro exemplo analisado em [Zan] com coeficientes variáveis foi:

$$
P=-\frac{\partial^{2}}{\partial x^{2}}-\phi^{2}(x) \frac{\partial^{2}}{\partial y^{2}}+a(x) \frac{\partial}{\partial y}
$$

onde $\phi, a \in C^{\infty}\left(T^{1}\right)$ a valores reais e são tais que:

1. $\phi(0) \neq 0$

2. $\phi(x)=0$, se $x \in[-\pi,-\pi / 2] \cup[\pi / 2, \pi]$

E o resultado obtido foi que $\mathrm{P}$ é $(\mathrm{GH}) .^{2}$

\subsection{Os espaços de Sobolev}

Definição 1.11 Para cada $s \in \mathbf{R}$, definimos:

$$
H^{s}\left(T^{n}\right)=\left\{u \in \mathcal{D}^{\prime}\left(T^{n}\right): \sum_{k \in Z^{n}}\left(1+|k|^{2}\right)^{s}|\hat{u}(k)|^{2}<\infty\right\}
$$

Podemos definir um produto interno em $H^{s}\left(T^{n}\right)$ por:

$$
<u, v>_{s}=\sum_{k \in \mathbf{Z}^{n}}\left(1+|k|^{2}\right)^{s} \hat{u}(k) \cdot \overline{\hat{v}(k)}
$$

\footnotetext{
${ }^{2}$ Referências: [Berg]; [Hou]; [Zan]
} 
e a norma associada a este produto é:

$$
\|u\|_{s}=\left\langle u, u>_{s}^{1 / 2}=\left[\sum_{k \in Z^{n}}\left(1+|k|^{2}\right)^{s}|\hat{u}(k)|^{2}\right]^{1 / 2}\right.
$$

Da desigualdade de Schwarz segue que:

$$
\left|\sum_{k}\left(1+|k|^{2}\right)^{(s+t) / 2} \hat{u}(k) \cdot \overline{\hat{v}(k)}\right|^{2} \leq\left(\sum_{k}\left(1+|k|^{2}\right)^{s}|\hat{u}(k)|^{2}\right)\left(\sum_{k}\left(1+|k|^{2}\right)^{t}|\hat{v}(k)|^{2}\right) .
$$

e então $\langle u, v\rangle_{s}$, existe se $u \in H^{t}$ e $v \in H^{t^{\prime}}$, onde $\left(t+t^{\prime}\right) / 2=s$. Com isto, temos que $H^{s}$ é um espaço de Hilbert.

Alguns resultados seguem do seguinte teorema:

Teorema 1.7 a) Seja $s \geq 0$ inteiro. Então existem constantes $C$ e $C$, dependendo no máximo de se $n$, tais que:

1. $C\|\phi\|_{s} \leq \sum_{|\alpha|=0}^{s}\left\|D^{\alpha} \phi\right\| \leq C^{\prime}\|\phi\|_{s}$ para todo $\phi \in C^{\infty}\left(T^{n}\right)$, onde $\|\cdot\|$ é a norma usual de $L^{2}\left(T^{n}\right)$.

Para o caso $s=0$, temos:

2. $\|\phi\|=\|\phi\|_{0}, \forall \phi \in C^{\infty}\left(T^{n}\right)$.

b) Se $t \leq s$, então $\|u\|_{t} \leq\|u\|_{s}$ e assim $H^{s} \subset H^{t}$.

c) $C^{\infty}\left(T^{n}\right)$ é um subespaço denso de $H^{s}\left(T^{n}\right)$ para todo s.

d) Se $u \in H^{s+t}$ e $v \in H^{s-t}$, então vale a desigualdade de Schwarz:

$$
\left\|<u, v>_{s}\right\| \leq\|u\|_{s+t}\|v\|_{s-t}
$$


e) $D^{\alpha}$ é um operador limitado de $H^{s+|\alpha|}$ em $H^{s}$ e para todo $s$, vale:

$$
\left\|D^{\alpha} u\right\|_{s} \leq\|u\|_{s+|\alpha|}, \forall u \in H^{s+|\alpha|} .
$$

Lema 1.1 (de Sobolev) Se $t \geq[n / 2]+1$ e $u \in H^{t}$, então a série: $\sum_{k} \hat{u}(k) e^{i x . k}$ converge uniformemente. Assim, a cada $u \in H^{t}\left(T^{n}\right)$, para $t \geq[n / 2]+1$, corresponde uma função contínua.

Corolário 1.1 Se $t \geq[n / 2]+1$, então $\exists c>0$ tal que se $\phi \in C^{\infty}\left(T^{n}\right)$, então:

$$
\|\phi\|_{\infty} \leq c\|\phi\|_{t} \text { e }\left\|D^{\alpha} \phi\right\|_{\infty} \leq c\|\phi\|_{t+[\alpha]} .
$$

Pode-se mostrar ainda que $\mathcal{D}^{\prime}=\cup_{s} H^{s}$ e $C^{\infty}=\cap_{s} H^{s}$. Esquematicamente, podemos escrever: se $s>0$, o operador $\wedge$ estabelece os isomorfismos:

$$
\begin{aligned}
& C^{\infty} \subset \ldots \subset H^{s} \subset \ldots \subset L^{2}=H^{0} \subset \ldots \subset H^{-s} \subset \ldots \subset \mathcal{D}^{\prime}
\end{aligned}
$$

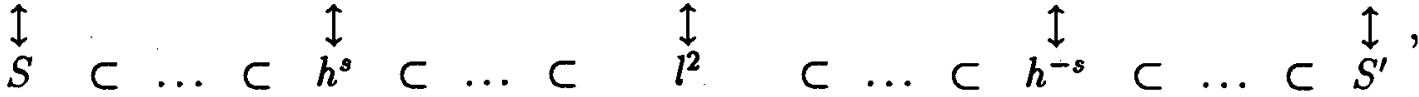

onde:

$$
l^{2}\left(\mathbf{Z}^{n}\right)=\left\{\left(a_{k}\right)_{k \in \mathbf{Z}^{n}} \in \mathbf{C} ; \sum_{k \in \mathbf{Z}^{n}}\left|a_{k}\right|^{2}<\infty\right\}
$$

e

$$
h^{s}\left(\mathbf{Z}^{n}\right)=\left\{\left(a_{k}\right)_{k \in Z^{n}} ; \sum_{k \in \mathbf{Z}^{n}}\left(1+|k|^{2}\right)^{s}\left|a_{k}\right|^{2}<\infty\right\}^{3} .
$$

\subsection{Alguns resultados de Análise}

\subsubsection{Teorema da aplicação aberta para espaços de Fréchet}

Sejam X,Y espaços de Fréchet, i.e., espaços vetoriais topológicos cujas respectivas topologias são definidas por métricas que os tornam completos. Seja $f: X \rightarrow Y$ linear, contínua e sobrejetora. Então $f$ é aberta ${ }^{4}$.

\footnotetext{
${ }^{3}$ Referência: [War]

${ }^{4}$ Referência: [Rud]
} 


\subsubsection{Operadores lineares contínuos e semi-normas}

Uma caracterização de operadores lineares contínuos definidos em espaços de Fréchet que será utilizada no capítulo 2 , é a seguinte:

Sejam $(X, d),\left(Y, d^{\prime}\right)$ espaços de Fréchet, cujas métricas $d$ e $d^{\prime}$ são definidas através de semi-normas $\left(p_{\alpha}\right)_{\alpha \in A} \mathrm{e}\left(q_{\beta}\right)_{\beta \in B}$, respectivamente. Então o operador linear $T: X \rightarrow Y$ é contínuo se, e somente se, $\forall \beta \in B$, $\exists F \subset A$ finito $e \exists c>0$ tais que:

$$
q_{\beta}(T \phi) \leq c \sum_{\alpha \in F} p_{\alpha}(\phi), \forall \phi \in X
$$

\subsubsection{Existência e regularidade de solução para uma E.D.O.}

Considere a equação: $a_{n}(t) x^{(n)}(t)+\cdots+a_{1}(t) x^{\prime}(t)+a_{0}(t) x(t)+b(t)=0$ com $a_{n}(t) \neq 0, \forall t$, e com valores iniciais: $x\left(t_{0}\right)=0, x^{\prime}\left(t_{0}\right)=0, \ldots, x^{(n-1)}\left(t_{0}\right)=1$.

$$
\begin{aligned}
& x(t)=x_{1}(t) \\
& x^{\prime}(t)=x_{1}^{\prime}(t)=x_{2}(t)
\end{aligned}
$$

Façamos : :

e teremos, na equação acima:

$$
x^{(n-1)}(t)=x_{n-1}^{\prime}(t)=x_{n}(t)
$$

$$
a_{n}(t) x_{n}^{\prime}(t)+a_{n-1}(t) x_{n}(t)+\cdots+a_{1}(t) x_{2}(t)+a_{0}(t) x_{1}(t)+b(t)=0 .
$$

Tais equações equivalem ao sistema linear:

$$
\begin{gathered}
X^{\prime}=A(t) X+h(t), \text { onde }: \\
X=\left[\begin{array}{c}
x \\
x^{\prime} \\
\vdots \\
x^{(n-1)}
\end{array}\right]=\left[\begin{array}{c}
x_{1} \\
x_{2} \\
\vdots \\
x_{n}
\end{array}\right] ; h=\left[\begin{array}{c}
0 \\
0 \\
\vdots \\
b
\end{array}\right] \\
A=\left[\begin{array}{ccccc}
0 & 1 & 0 & \ldots & 0 \\
0 & 0 & 1 & \ldots & 0 \\
\ldots & \ldots & \ldots & \ldots & \ldots \\
\frac{-a_{0}}{a_{n}} & \frac{-a_{1}}{a_{n}} & \frac{-a_{2}}{a_{n}} & \ldots & \frac{-a_{n-1}}{a_{n}}
\end{array}\right] .
\end{gathered}
$$


Segundo [Hale], existe uma única solução $X(t) \in C^{1}$ para o PVI ralativo ao sistema e, portanto, uma única solução $x(t) \in C^{n}$ para o PVI relativo à primeira equação, uma vez que $b, a_{0}, a_{1}, \ldots, a_{n} \in C^{\infty}$. E podemos ver que $x \in C^{\infty}$. (Basta voltar ao sistema anterior $\mathrm{e}$ observar que: se $x_{1}, x_{2}, \ldots, x_{n} \in C^{1}$, então $x_{n}^{\prime} \in C^{1} \Rightarrow x_{n}=x_{n-1}^{\prime} \in C^{2} \Rightarrow$ $x_{n-1} \in C^{3}, \ldots, x_{1} \in C^{n+1} \mathrm{e}$, prosseguindo indutivamente, concluímos que $\left.x \in C^{\infty}\right)$. 


\section{Capítulo 2}

\section{Hipoeliticidade e Resolubilidade Globais para Alguns Operadores a Coeficientes Constantes}

Neste capítulo serão utilizados os resultados e caracterizações do capítulo anterior, a fim de estudarmos alguns tipos de operadores diferenciais a coeficientes constantes.

As questões principais a serem resolvidas serão:

- dado um operador diferencial $P: C^{\infty}\left(T^{n}\right) \rightarrow C^{\infty}\left(T^{n}\right)$, sob que condições $P$ é sobrejetor, ou seja: para toda $g \in C^{\infty}\left(T^{n}\right)$, sempre existe $f \in C^{\infty}\left(T^{n}\right)$ tal que $P f=g$ ?

- Sob que condições a solução é $C^{\infty}$ ?

Eventualmente, pode-se perguntar quando é que $P$ é injetor, ou seja, quando é que a solução para a equação $P u=f$ é única.

Na primeira seção será vista uma generalização (feita em [Zan]) do artigo de [G-W] para o toro $n$-dimensional ( $n \geq 2$ ), o qual trata da hipoeliticidade global de alguns tipos de operadores (teor. 2.1) e sua relação com a resolubilidade no espaço $C^{\infty}\left(T^{n}\right)$ (corol. 2.1 ).

$\mathrm{Na}$ segunda seção, serão abordados resultados semelhantes aos anteriores vistos em [Mos], onde foram tratados de forma mais analitica, destacando-se aí a utilização do Teorema da Aplicação Aberta para Espaços de Fréchet. Neste caso, 
também foi feita uma generalização (teor. 2.2), e a análise de uma classe de operadores feita por [Mos] segue como corolário (teor.2.3).

Com base nestes resultados, na terceira seção serão estudados exemplos de operadores globalmente hipoelíticos (GH) e globalmente resolúveis (GR), bem como outros exemplos que não apresentam tais propriedades.

\subsection{Hipoeliticidade Global e Resolubilidade em $T^{n}$}

Introduzimos aqui o conceito de operador globalmente resolúvel que será utilizado no decorrer deste capítulo:

Definição 2.1 Seja o operador diferencial linear $P: X \rightarrow X$, com $X \subset \mathcal{D}^{\prime}\left(T^{n}\right)$ um subespaço. Então $P$ será dito globalmente resolúvel (GR) em $X$, se for uma aplicação sobrejetora.

Consideremos o operador diferencial $P$ em $T^{n}, n \geq 2$ :

$$
\begin{gathered}
P=\sum_{|\alpha| \leq m} c_{\alpha} D_{1}^{\alpha_{1}} \ldots D_{n}^{\alpha_{n}}, \text { onde } D_{j}=\frac{1}{i} \frac{\partial}{\partial x_{j}} \\
c_{\alpha} \in \mathbf{C} ; \quad \alpha=\left(\alpha_{1}, \ldots, \alpha_{n}\right) \in \mathbf{Z}_{+}^{n} \text { e }|\alpha|=\alpha_{1}+\cdots+\alpha_{n} .
\end{gathered}
$$

$\operatorname{Definimos} P(k)=\sum_{|\alpha| \leq m} c_{\alpha} k_{1}^{\alpha_{1}} \ldots k_{n}^{\alpha_{n}}$, onde $k=\left(k_{1}, \ldots, k_{n}\right) \in \mathbf{Z}^{n}$.

Teorema 2.1 $P$ definido como acima é (GH) se, e somente se, existem números reais positivos $L, M$ tais que:

(LM) $\quad|P(k)| \geq \frac{L}{\left(k_{1}^{2}+\cdots+k_{n}^{2}\right)^{M}}$, para $|k|$ suficientemente grande. 
Demonstração: Suponhamos que existam r,L e M positivos tais que:

$$
|P(k)| \geq \frac{L}{|k|^{2 M}} \text { para }|k|=\left(k_{1}^{2}+\cdots+k_{n}^{2}\right)^{1 / 2} \geq r .
$$

Assim, $P(k)=0$ implica que $k \in A=\left\{k \in \mathbf{Z}^{n} ;|k|<r\right\}$, o qual é finito. Seja $f=\sum_{k \in Z^{n}} \hat{f}(k) e_{k} \in \mathcal{D}^{\prime}\left(T^{n}\right)$, onde $e_{k}(x)=e^{i k . x}$, tal que $P f=g \in C^{\infty}\left(T^{n}\right)$.

Então, temos: $\hat{f}(k) \cdot P(k)=\hat{g}(k), k \in \mathbf{Z}^{n}$.

Dado $\mathrm{N} \in \mathrm{N}$, para todo $k \in \mathbf{Z}^{n}$ com $|k| \geq r$, existe $C>0$ tal que:

$$
|\hat{f}(k)|=\left|\frac{\hat{g}(k)}{P(k)}\right| \leq|\hat{g}(k)| \cdot \frac{|k|^{2 M}}{L} \leq C|k|^{-\mathrm{N}}
$$

pois $(\hat{g}(k))$ decresce rapidamente. Logo,

$$
\sup \left\{|\hat{f}(k) \| k|^{\mathrm{N}} ; k \in \mathbf{Z}^{n}\right\} \leq \max _{0 \leq|k|<r}\left\{|\hat{f}(k) \| k|^{\mathrm{N}}, C\right\}<\infty .
$$

Portanto, $f \in C^{\infty}\left(T^{n}\right)$ e concluímos que $P$ é (GH). Suponha, agora, que a condição (LM) seja falsa. Então existe uma sequência $\left(k_{j}\right) \in \mathbf{Z}^{n}$ tal que:

$$
\left|P\left(k_{j}\right)\right| \leq \frac{1}{\left|k_{j}\right|^{2 j}} e\left|k_{j}^{i}\right| \rightarrow \infty, \forall i=1, \ldots, n
$$

Defina $f=\sum_{j=1}^{\infty} e_{k_{j}}$. Como

$$
\hat{f}(k)= \begin{cases}1 & \text { se } k=k_{j} \text { para algum } j \in \mathbf{N} \\ 0 & \text { se } k \neq k_{j}, j \in \mathbf{N}\end{cases}
$$

temos que $f \in \mathcal{D}^{\prime}\left(T^{n}\right)$. Além disso, $f \notin C^{\infty}\left(T^{n}\right)$, uma vez que a subsequência $\left(\hat{f}\left(k_{j}\right)\right)$ não decresce rapidamente.

Mas:

$$
P f=\sum_{k \in \mathbf{Z}^{n}} \hat{f}(k) P(k) e_{k}=\sum_{j=1}^{\infty} P\left(k_{j}\right) e_{k_{j}}=g,
$$

com

$$
\hat{g}(k)= \begin{cases}0 & \text { se } k \neq k_{j}, j \in \mathbf{N} \\ P\left(k_{j}\right) & \text { se } k=k_{j} \text { para algum } j \in \mathbf{N}\end{cases}
$$


Logo, para todo $k \in \mathbf{Z}^{n}, k \neq 0 e \mathbf{N} \in \mathbf{N}$, temos:

$$
|\hat{g}(k)| \leq\left|P\left(k_{j}\right)\right| \leq \frac{1}{\left|k_{j}\right|^{2 j}} \leq C \frac{1}{|k|^{\mathrm{N}}}
$$

e, portanto, $g \in C^{\infty}\left(T^{n}\right)$, o que mostra que $P$ não é (GH).

Um resultado interessante segue do teorema anterior e trata da resolubilidade de $P$ em $C^{\infty}\left(T^{n}\right)$, a menos de um espaço de dimensão finita:

Corolário 2.1 Se $P$ é (GH), então $P$ é Fredholm de indice zero, i.e., $\operatorname{dim} \operatorname{Ker} P=\operatorname{codim} \operatorname{Im} P$.

Prova: $P: C^{\infty}\left(T^{n}\right) \rightarrow C^{\infty}\left(T^{n}\right)$ é contínuo, uma vez que a métrica em $C^{\infty}\left(T^{n}\right)$ envolve derivadas de todas as ordens. Como $P$ é $(\mathrm{GH})$, então vale a condição (LM) do teorema anterior, a qual implica que $P(k)$ se anula apenas para um conjunto finito de índices: $S=\left\{k \in \mathbf{Z}^{n} ; P(k)=0\right\}$. Se $f \in C^{\infty}\left(T^{n}\right)$ considere $\pi_{S} f$ a função definida por:

$$
\pi_{S} f=\sum_{k \in \mathbb{Z}^{n}} a_{k} e_{k}
$$

onde

$$
a_{k}= \begin{cases}\hat{f}(k) & \text { se } k \in S \\ 0 & \text { se } k \notin S\end{cases}
$$

Como $S$ é finito, $\pi_{S}$ é uma projeção contínua. Além disso, temos:

$$
\begin{gathered}
\operatorname{Ker} P=\left\{f \in C^{\infty}\left(T^{n}\right): \hat{f}(k)=0, \forall k \in \mathbf{Z}^{n} \backslash S\right\}= \\
=\left\{f \in C^{\infty}\left(T^{n}\right):\left(I-\pi_{S}\right) f=0\right\}=\left\{f \in C^{\infty}\left(T^{n}\right): f=\pi_{S} f\right\} .
\end{gathered}
$$

Afirmação: Se $g \in C^{\infty}\left(T^{n}\right)$ é tal que $\pi_{S} g=0$, então existe $f \in C^{\infty}\left(T^{n}\right)$ tal que $P f=g$.

De fato: defina $f=\sum_{k} \hat{f}(k) \cdot e_{k}$, onde

$$
\hat{f}(k)= \begin{cases}\frac{\hat{g}(k)}{P(k)} & \text { se } k \in \mathbf{Z}^{n} \backslash S \\ 0 & \text { se } k \in S\end{cases}
$$


e a condição (LM) garante o decrescimento rápido de $(\hat{f}(k))_{k \in Z^{n}}$. Além disso, $P(k) \hat{f}(k)=0 . \hat{f}(k)=0=\hat{g}(k)$, se $k \in S$ e $P(k) \hat{f}(k)=\hat{g}(k)$, se $k \in \mathbf{Z}^{n} \backslash S$.

Esta última afirmação equivale a dizer que $\operatorname{Ker}\left(\pi_{S}\right) \subset \operatorname{ImP}$ e é claro que $\operatorname{Im} P \subset \operatorname{ker}\left(\pi_{s}\right)$.

Temos: $\operatorname{dim} \operatorname{Ker} P=$ cardinalidade de $S<\infty$.

Mostremos que $C^{\infty}\left(T^{n}\right)=\operatorname{ImP} \oplus \operatorname{Ker} P$, donde concluiremos que $\operatorname{dim} \operatorname{Ker} P=\operatorname{codim} \operatorname{Im} P=\# S$.

Com efeito,

$$
\operatorname{Ker} P=\left[e_{k}: k \in S\right] \text { e } \operatorname{Im} P=\operatorname{Ker}\left(\pi_{S}\right)
$$

Logo:

$$
\operatorname{Ker} P \cap \operatorname{Im} P=\operatorname{Ker} P \cap \operatorname{Ker}\left(\pi_{S}\right)=\{0\}
$$

Além disso, $K \operatorname{er} P$ e $\operatorname{Im} P$ são subespaços fechados de $C^{\infty}\left(T^{n}\right)$, pois $P$ e $\pi_{S}$ são aplicações contínuas.

Assim, $\forall f \in C^{\infty}\left(T^{n}\right)$, podemos escrever:

$$
f=\left(I-\pi_{S}\right) f+\pi_{S} f
$$

onde a primeira parcela está em $\operatorname{Ker}\left(\pi_{S}\right)$ e a segunda está em $K e r P$, uma vez que :

$$
\pi_{S} \circ\left(I-\pi_{S}\right) f=\left(\pi_{S}-\pi_{S}^{2}\right) f=0
$$

\subsection{Resolubilidade em $C_{S}^{\infty}\left(T^{n}\right)$}

Seja $P$ um operador diferencial linear a coeficientes constantes como na seção anterior. Fazemos:

$$
S=\left\{k \in \mathbf{Z}^{n} ; P(k)=0\right\}
$$

. Se $\# S<\infty$, definimos, para cada $f=\sum_{k \in Z^{n}} \hat{f}(k) e_{k} \in \mathcal{D}^{\prime}\left(T^{n}\right)$, a função: 


$$
\pi_{S} f=\sum_{k \in S} \hat{f}(k) e_{k}
$$

onde $\pi_{S}: \mathcal{D}^{\prime}\left(T^{n}\right) \rightarrow \mathcal{D}^{\prime}\left(T^{n}\right)$ é uma projeção sobre o subespaço: $H_{S}=\left[e_{k}: k \in S\right]$ e $e_{k}(x)=e^{i k . x}, x \in T^{n}$.

\section{Definimos :}

$$
C_{S}^{\infty}\left(T^{n}\right)=\left\{f-\pi_{S} f ; f \in C^{\infty}\left(T^{n}\right)\right\},
$$

cuja topologia será a induzida de $C^{\infty}\left(T^{n}\right)$. Então, vale um resultado equivalente ao teorema (2.1):

Teorema 2.2 $P: C_{S}^{\infty}\left(T^{n}\right) \rightarrow C_{S}^{\infty}\left(T^{n}\right) e^{e}$ uma bijeção se, e somente se, vale a condição:

$$
|P(k)| \geq C(1+|k|)^{-\tau_{0}} \text { para algum } C>0 \text { e } \tau_{0}>0, \forall k \in \mathbf{Z}^{n} \backslash S .
$$

Observemos que o subspaço $C_{S}^{\infty}\left(T^{n}\right)$ é colocado no dominio a fim de garantir a injetividade do operador $P$ e, no contra-domínio, para possibilitar que $P$ seja sobrejetor, uma vez que as funções $f \in\left[e_{k}: k \in S\right]$ não são atingidas pelo operador $P$, i.e., não estão em $\operatorname{Im} P$. De fato: se $f=\sum_{j=1}^{r} \beta_{j} e_{k_{j}}$, com $k_{j} \in$ $S$ e $\beta_{j} \neq 0$, teremos:

$\forall u \in \mathcal{D}^{\prime}\left(T^{n}\right): P\left(k_{j}\right) \hat{u}\left(k_{j}\right)=0 \neq \beta_{j}=\hat{f}\left(k_{j}\right)$.

Ainda, vale ressaltar que a condição (2.1) é semelhante à condição (LM) do teorema (2.1), mas, como foi observado no início deste capítulo, aqui será tratada de forma mais analítica, embora seja válida uma demonstração análoga à do teorema da seção anterior.

Demonstração: $O$ espaço $C_{S}^{\infty}\left(T^{n}\right)$ é um espaço de Fréchet cuja topologia pode ser descrita pelas normas de Sobolev:

$$
\|\varphi\|_{r}=\left[\sum_{k \in \mathbb{Z}^{n}}(1+|k|)^{2 r}|\hat{\varphi}(k)|^{2}\right]^{1 / 2}
$$

onde:

$$
\varphi(x)=\sum_{k \in Z^{n}} \hat{\varphi}(k) e_{k}, \text { e } \hat{\varphi}(k)=0 \text { se } k \in S
$$


Supondo $P$ bijetor, pelo Teorema da Aplicação Aberta (para Espaços de Fréchet), temos que $P^{-1}$ é contínuo. Da definição (1.5.2) segue, em particular, que existem constantes $\tau, c_{1}>0$ tais que:

$$
\|\varphi\|_{0} \leq c_{1}\|P \varphi\|_{2 \tau}, \forall \varphi \in C_{S}^{\infty}\left(T^{n}\right),
$$

a qual afirmamos ser equivalente a :

$$
(1+|k|)^{-2 \tau} \leq c_{1}|P(k)|, \forall k \in \mathbf{Z}^{n} \backslash S
$$

De fato:

- $(2.2) \Rightarrow(2.3)$ :

Dado $k \in \mathbf{Z}^{n} \backslash S$, definimos $\varphi_{k}(x)=e^{i k . x}$ e temos:

$$
\left|\hat{\varphi_{k}}(k)\right|=1 ;\left|\hat{\varphi_{k}}(j)\right|=0 \text { se } j \neq k
$$

Logo, $\left\|\varphi_{k}\right\|_{0}=1$ e então:

$$
\begin{aligned}
& \quad\left\|P \varphi_{k}\right\|_{2 \tau}=\left[\sum_{j \in \mathbf{Z}^{n}}(1+|j|)^{4}|P(j)|^{2}|\hat{\varphi}(j)|^{2}\right]^{1 / 2}=(1+|k|)^{2 \tau}|P(k)| \stackrel{(2.2)}{\Rightarrow} \\
& \stackrel{(2.2)}{\Rightarrow} 1 \leq c_{1}(1+|k|)^{2 \tau}|P(k)| \Rightarrow(1+|k|)^{-2 \tau} \leq c_{1}|P(k)| \\
& \text { - }(2.3) \Rightarrow(2.2):
\end{aligned}
$$

$$
\begin{aligned}
c_{1}^{2}\|P \varphi\|_{2 \tau}^{2} & =c_{1}^{2} \sum_{k \in \mathbb{Z}^{n}}(1+|k|)^{4 \tau}|P(k)|^{2}|\hat{\varphi}(k)|^{2} \\
& \geq c_{1}^{2} \sum_{k \in Z^{n}}(1+|k|)^{4 \tau} \frac{(1+|k|)^{-4 \tau}}{c_{1}^{2}}|\hat{\varphi}(k)|^{2} \\
& =\sum_{k \in Z^{n}}|\hat{\varphi}(k)|^{2}=\|\varphi\|_{0}^{2} .
\end{aligned}
$$


Finalmente, de (2.3) segue a desigualdade (2.1), tomando-se: $c=1 / c_{1} \mathrm{e}$ $\tau_{0}=2 \tau>0$.

Reciprocamente, se vale (2.1), definamos para $f \in C_{S}^{\infty}\left(T^{n}\right)$ a função:

$$
u(x)=\sum_{k \in \mathbf{Z}^{n} \backslash S} \frac{\hat{f}(k)}{P(k)} e^{i k . x} .
$$

Temos que $u \in C_{S}^{\infty}\left(T^{n}\right)$, pois $\hat{u}(k)=\hat{f}(k)=0$ se $k \in S$ e

$$
|\hat{u}(k)|=\frac{|\hat{f}(k)|}{|P(k)|} \leq \frac{|\hat{f}(k)|}{c(1+|k|)^{-\tau_{0}}}, \forall k \in \mathbf{Z}^{n} \backslash S
$$

e como $f \in C_{S}^{\infty}\left(T^{n}\right)$, segue que $u \in C_{S}^{\infty}\left(T^{n}\right)$ e além disso, $u$ é solução para a equação $P u=f$.

Como caso particular deste teorema, segue o resultado visto em [Mos]:

Teorema $2.3 O$ operador $L=\sum_{j=1}^{n} \tilde{D}_{j}{ }^{2}: C_{0}^{\infty}\left(T^{n+1}\right) \rightarrow C_{0}^{\infty}\left(T^{n+1}\right)$, onde $\tilde{D}_{j}=\partial x_{j}-\alpha_{j} \partial x_{n+1}, \quad j=1, \ldots, n$, é uma bijeção se, e somente se, $\alpha=\left(\alpha_{1}, \ldots, \alpha_{n}\right) \in \mathbf{R}^{n} \backslash \mathbf{Q}^{n}$ satisfaz a condição Diofantina:

$$
\begin{gathered}
\sum_{j=1}^{n}\left(\alpha_{j} k_{n+1}-k_{j}\right)^{2} \geq c_{0}^{-1}\left(1+k_{n+1}^{2}\right)^{-\tau} \text { para } c_{0}, \tau \geq 0 e \\
\forall k=\left(k_{1}, \ldots, k_{n}, k_{n+1}\right) \in \mathbf{Z}^{n+1} \backslash\{0\}
\end{gathered}
$$

Demonstração: Neste caso, é conveniente tomar em $C_{0}^{\infty}=C_{S}^{\infty}\left(T^{n+1}\right)$ com $S=\{0\}$, a topologia descrita pelas normas de Sobolev abaixo, a qual coincide com as normas tomadas no teorema $(2.2)^{1}$ :

$$
\|\varphi\|_{r}=\left[\sum_{k \in \mathbf{Z}^{n+1}}|k|^{2 r}|\hat{\varphi}(k)|^{2}\right]^{1 / 2}
$$

\footnotetext{
${ }^{1}$ ver Apêndice
} 
onde

$$
\varphi=\sum_{k} \hat{\varphi}(k) e_{k} \text { e } \hat{\varphi}(0)=0
$$

Como $L$ é bijeção, da mesma maneira que no teorema anterior, concluímos que existem constantes $\tau, c_{1}>0$ tais que:

$$
\|\varphi\|_{0} \leq c_{1}\|L \varphi\|_{2 \tau}, \forall \varphi \in C_{0}^{\infty}\left(T^{n+1}\right)
$$

a qual é equivalente à condição:

$$
|k|^{-2 \tau} \leq c_{1} \sum_{j=1}^{n}\left(k_{j}-\alpha_{j} k_{n+1}\right)^{2}=c_{1}|L(k)|, \forall k \in \mathbf{Z}^{n+1} \backslash\{0\}
$$

Afirmação: estas desigualdades implicam (2.4), que é aparentemente mais forte, com $c_{0}=\max \left\{2, c_{1}\left(2|\alpha|^{2}+1\right)^{\tau}\right\}$ e $|\alpha|^{2}=\alpha_{1}^{2}+\cdots+\alpha_{n}^{2}$.

De fato:

- Se $|\bar{k}|^{2}=k_{1}^{2}+\cdots+k_{n}^{2} \geq 2|\alpha|^{2} k_{n+1}^{2}+1$, então:

$$
\begin{aligned}
\sum_{j=1}^{n}\left(k_{j}-\alpha_{j} k_{n+1}\right)^{2} & \geq|\bar{k}|^{2}-2\left|k_{n+1}\right|\left|\sum_{j} \alpha_{j} k_{j}\right|+k_{n+1}^{2}|\alpha|^{2} \\
& \geq|\bar{k}|^{2}-2\left|k_{n+1}\right||\alpha||\bar{k}|+k_{n+1}^{2}|\alpha|^{2}=\left(|\bar{k}|-\left|k_{n+1}\right||\alpha|\right)^{2} \\
& \geq \frac{1}{2}|\bar{k}|^{2}-|\alpha|^{2} k_{n+1}^{2} \geq|\alpha|^{2} k_{n+1}^{2}+\frac{1}{2}-|\alpha|^{2} k_{n+1}^{2}=\frac{1}{2}
\end{aligned}
$$

Assim, (2.4) é trivialmente satisfeita com $c_{0}=2$ e $\tau=0$.

- Se $|\bar{k}|^{2} \leq 2|\alpha|^{2} k_{n+1}^{2}+1$, então:

$$
|k|^{2}=|\bar{k}|^{2}+k_{n+1}^{2} \leq 2|\alpha|^{2} k_{n+1}^{2}+1+k_{n+1}^{2} \leq\left(2|\alpha|^{2}+1\right)\left(k_{n+1}^{2}+1\right),
$$

de modo que (2.6) implica (2.4), com $c_{0} \leq c_{1}\left(2|\alpha|^{2}+1\right)^{\tau}$. 
Reciprocamente, a condição (2.4) implica que $L: C_{0}^{\infty} \rightarrow C_{0}^{\infty}$ é bijeção (- a demonstração é análoga ao que foi feito em (2.2), usando a representação em série de Fourier).

Observemos que o teorema (2.2) e o corolário (2.1) nos dizem que, se $P$ é (GH), então $P$ é (GR) em $C^{\infty}\left(T^{n}\right)$, a menos de um espaço de dimensão finita. Segue, então, o resultado:

Corolário $2.2 P$ é $(G R)$ em $C^{\infty}\left(T^{n}\right)$ se, e somente se, ocorrem as duas condições abaixo:

1. $P \dot{e}(G H) e m T^{n}$.

2. $P(k) \neq 0, \forall k \in \mathbf{Z}^{n}$.

Prova: Suponha que $P$ seja (GR) em $C^{\infty}\left(T^{n}\right)$. Então, $P: C^{\infty}\left(T^{n}\right) \rightarrow C^{\infty}\left(T^{n}\right)$ é sobrejetor.

Provemos 2): suponha que exista $k_{0} \in S=\left\{k \in \mathbf{Z}^{n}: P(k)=0\right\}$. Tome $f(x)=e^{i k_{0} \cdot x}$. Como $P$ é sobre, existe $u \in C^{\infty}\left(T^{n}\right)$ tal que $P u=f$. Logo:

$P\left(k_{0}\right) \hat{u}\left(k_{0}\right)=\hat{f}\left(k_{0}\right)=1$. Mas $P\left(k_{0}\right)=0$ ! (Absurdo!)

Concluímos, então, que $P(k) \neq 0, \forall k \in \mathbf{Z}^{n}$.

Afirmação: $P$ é injetor.

De fato: suponha $v \in K e r P$, i.e., $P v=0 \Rightarrow P(k) v(k)=0 \Rightarrow$ $\hat{v}(k)=0, \forall k \Rightarrow v=0$.

Pelo teorema (2.2), para todo $k \in \mathbf{Z}^{n}$, vale:

$$
|P(k)| \geq C(1+|k|)^{-\tau_{0}}, \text { para alguma constante } c>0 \text { e } \tau_{0}>0,
$$

o que equivale à condição (LM) de (2.1), i.e., existem constantes $L, M>0$ tais que:

$$
|P(k)| \geq \frac{L}{|k|^{M}}, \text { para }|k| \text { suficientemente grande. }
$$


Portanto, $P$ é $(\mathrm{GH})$ e assim, para todo $u \in \mathcal{D}^{\prime}\left(T^{n}\right)$ tal que $P u=f \in C^{\infty}\left(T^{n}\right)$, temos que $u \in C^{\infty}\left(T^{n}\right)$.

Reciprocamente, se valem (1) e (2), então $|P(k)| \geq \frac{L}{|k|^{M}}$ para $|k|$ suficientemente grande (digamos, $|k| \geq R>0)$ e como $P(k) \neq 0, \forall k$, existem constantes $C>0$ e $\tau_{0}>0$ tais que:

$$
|P(k)| \geq C(1+|k|)^{-\tau_{0}}, \forall k \in \mathbf{Z}^{n} .
$$

De fato: para $|k| \geq R$, são equivalentes:

$$
|P(k)| \geq \frac{L}{|k|^{M}}
$$

e

$$
|P(k)| \geq \frac{L_{1}}{(1+|k|)^{M}}, M>0, \text { onde } L, L_{1} \text { são positivos. }
$$

Seja o conjunto $A=\left\{k \in \mathbf{Z}^{n} ;|k|<R\right\}=\left\{k^{(1)}, \ldots, k^{(s)}\right\}$.

Tome

$$
0<C<\min \left\{L_{1},\left|P\left(k_{j}\right)\right|\left(1+\left|k^{(j)}\right|\right)^{\tau_{0}} ; j=1, \ldots, s\right\}
$$

e teremos :

$$
\left|P\left(k^{(j)}\right)\right| \geq C\left(1+\left|k^{(j)}\right|\right)^{\tau_{0}}, j=1, \ldots, s .
$$

Concluímos, pelo teorema(2.2), que $P: C^{\infty}\left(T^{n}\right) \rightarrow C^{\infty}\left(T^{n}\right)$ é uma bijeção, i.e, $P$ é (GR) em $T^{n}$.

Com isso, vemos que o operador $L$ do teorema (2.3) não é (GR) em $C^{\infty}\left(T^{n+1}\right)$.

\subsection{Exemplos de operadores (GH) e operado- res (GR)}

Um exemplo interessante a ser analisado envolve o conceito de número de Liouville: 
Definição $2.2 U m$ número irracional $\alpha$ é de Liouville se, para todo $N \in \mathrm{N}$, existem $K>0$ e uma infinidade de pares $(n, m) \in \mathbf{Z}^{2}$ tais que:

$$
\left|\alpha-\frac{n}{m}\right|<\frac{K}{m^{N}}
$$

Consideremos o operador $P: C^{\infty}\left(T^{2}\right) \rightarrow C^{\infty}\left(T^{2}\right)$, dado por:

$$
P=D_{1}-\alpha D_{2}, \text { onde } D_{j}=\frac{1}{i} \frac{\partial}{\partial x_{j}}, j=1,2 \text {. }
$$

Teorema 2.4 a) Se $\alpha=a+i b, a, b \in \mathbf{R}, b \neq 0$, então $P$ é $(G H)$ em $T^{2}$.

b) Se $\alpha \in \mathbf{Q}$, então $P$ não é (GH).

c) Se $\alpha \in \mathbf{R} \backslash \mathbf{Q}$, então $P$ é $(G H)$ se, e somente se, $\alpha$ é não-Liouville.

Demonstração: a) Para todo $(m, n) \in \mathbf{Z}^{2}$ tal que $|n| \geq 1 /|b|$, temos :

$|P(m, n)|^{2}=|m-(a+i b) n|^{2}=|m-a n+i b n|^{2}=(m-a n)^{2}+b^{2} n^{2} \geq b^{2} n^{2} \geq 1$.

Pelo teorema (2.1), $P$ é (GH).

b) Seja $\alpha=p / q, p, q \in \mathbf{Z}, q \neq 0$. Então, para qualquer inteiro $\mathrm{m}$, temos:

$$
P(m p, m q)=m p-\frac{p}{q} \cdot m q=0
$$

ou seja, $P$ tem uma infinidade de zeros inteiros e, pelo teorema (2.1), segue que $P$ não é (GH).

c) Se $\alpha \in \mathbf{R} \backslash \mathbf{Q}$ é de Liouville, então, para todo $M \in \mathbf{N}$ existe $L>0$ e uma sequência $\left(m_{j}, n_{j}\right) \in \mathbf{Z}^{2}$, onde podemos supor $m_{j}>0$ e $m_{j} \rightarrow \infty$, tais que:

$$
\left|\alpha-\frac{n_{j}}{m_{j}}\right|<\frac{L}{m_{j}^{M}}
$$


Logo, $\left|P\left(n_{j}, m_{j}\right)\right|=\left|\alpha m_{j}-n_{j}\right|<L / m_{j}^{M-1}$

Como $m_{j} \rightarrow \infty$, supomos $L / m_{j}^{M-1}<1$.

Assim:

$$
\left|n_{j}\right|-|\alpha| m_{j} \leq\left|\alpha m_{j}-n_{j}\right|<\frac{L}{m_{j}^{M-1}}=m_{j} \frac{L}{m_{j}^{M}}<m_{j} .
$$

Portanto, $\left|n_{j}\right| \leq(1+|\alpha|) m_{j}$, donde:

$$
\begin{gathered}
n_{j}^{2}+m_{j}^{2} \leq\left[1+(1+|\alpha|)^{2}\right] m_{j}^{2} \\
\left(n_{j}^{2}+m_{j}^{2}\right)^{\frac{M-1}{2}} \leq\left[1+(1+|\alpha|)^{2}\right]^{\frac{M-1}{2}} m_{j}^{M-1}
\end{gathered}
$$

e

$$
\frac{1}{m_{j}^{M-1}} \leq \frac{\left[1+(1+|\alpha|)^{2}\right]^{\frac{M-1}{2}}}{\left(n_{j}^{2}+m_{j}^{2}\right)^{\frac{M-1}{2}}}
$$

De $\left({ }^{*}\right)$, segue que:

$$
\left|P\left(n_{j}, m_{j}\right)\right|<\frac{L\left[1+(1+|\alpha|)^{2}\right]^{\frac{M-1}{2}}}{\left(m_{j}^{2}+n_{j}^{2}\right)^{\frac{M-1}{2}}} ;
$$

escolhemos, então, $L^{\prime}, M^{\prime}$ tais que:

$$
\frac{L\left[1+(1+|\alpha|)^{2}\right]^{\frac{M-1}{2}}}{\left(m_{j}^{2}+n_{j}^{2}\right)^{\frac{M-1}{2}}}<\frac{L^{\prime}}{\left(m_{j}^{2}+n_{j}^{2}\right)^{M^{\prime}}}
$$

e pelo teorema(2.1), $P$ não é (GH).

Suponhamos, agora, que $P$ não seja (GH).

Então, para todo $L>0$ e $j \in \mathbf{N}$, existe $\left(n_{j}, m_{j}\right) \in \mathbf{Z}^{2}$ tal que $\left|n_{j}\right| \rightarrow \infty, m_{j}>0$ e $m_{j} \rightarrow \infty$ quando $j \rightarrow \infty$ e

$$
\left|P\left(n_{j}, m_{j}\right)\right|<\frac{L}{\left(n_{j}^{2}+m_{j}^{2}\right)^{j}} \leq \frac{L}{m_{j}^{2 j}} .
$$

Assim:

$$
\left|\alpha m_{j}-n_{j}\right|<\frac{L}{m_{j}^{2 j}} \Rightarrow
$$




$$
\left|\alpha-\frac{n_{j}}{m_{j}}\right|<\frac{L}{m_{j}^{2 j-1}}
$$

ou seja, $\alpha$ é de Liouville.

Observação 2.1 Em [Zan] foi visto o seguinte resultado:

$$
\text { Se } P=a_{0}\left(\frac{\partial}{\partial x}-\alpha_{1} \frac{\partial}{\partial y}\right) \ldots\left(\frac{\partial}{\partial x}-\alpha_{m} \frac{\partial}{\partial y}\right), \operatorname{com} a_{0} \in \mathbf{C}, a_{0} \neq 0
$$

então $P$ é (GH) se, e somente se, $\alpha_{i}$ é irracional não-Liouville, para todo $i=1, \ldots, m$.

Assim, o operador $P=\left(D_{1}-\alpha D_{2}\right)^{2}$ é um exemplo que ilustra este resultado, se $\alpha \in \mathbf{R} \backslash \mathbf{Q}$ é não-Liouville. A este mesmo operador se aplica o teorema (2.3), com $n=1$, ou seja, $P: C_{0}^{\infty}\left(T^{2}\right) \rightarrow C_{0}^{\infty}\left(T^{2}\right)$ é uma bijeção se, e somente se, existem constantes $c_{0}>0$ e $\tau_{0}>0$ tais que:

$$
\left(\alpha k_{2}-k_{1}\right)^{2} \geq c_{0}^{-1}\left(1+k_{2}^{2}\right)^{-\tau_{0}}, \forall\left(k_{1}, k_{2}\right) \in \mathbf{Z}^{2} \backslash\{0\}
$$

o que é equivalente a $\alpha$ ser um irracional não-Liuoville.

Com efeito,

$$
k_{2}^{2}\left(\alpha-\frac{k_{1}}{k_{2}}\right)^{2} \geq c_{0}^{-1}\left(1+k_{2}^{2}\right)^{-\tau_{0}} \Leftrightarrow\left(\alpha-\frac{k_{1}}{k_{2}}\right)^{2} \geq c_{0}^{-1} \frac{\left(1+k_{2}^{2}\right)^{-\tau_{0}}}{k_{2}^{2}}
$$

Mostremos que (2.7) $\Leftrightarrow \alpha$ é não-Liouville:

$$
\begin{gathered}
\left(\alpha-\frac{k_{1}}{k_{2}}\right)^{2} \geq c_{0}^{-1} \frac{\left(1+k_{2}^{2}\right)^{-\tau_{0}}}{\left(1+k_{2}^{2}\right)^{2}}=c_{0}^{-1}\left(1+k_{2}^{2}\right)^{-\left(\tau_{0}+2\right)} \\
\Rightarrow\left|\alpha-\frac{k_{1}}{k_{2}}\right| \geq c_{0}^{-1 / 2}\left(1+k_{2}^{2}\right)^{-\left(\tau_{0}+2\right) / 2}
\end{gathered}
$$

E, para $k_{2} \in \mathbf{Z} ; k_{2}^{2} \geq 1$, temos:

$$
1+k_{2}^{2} \leq 2 k_{2}^{2} \Rightarrow\left(1+k_{2}^{2}\right)^{-1} \geq 2^{-1} \cdot k_{2}^{-2} .
$$


Logo:

$$
\left|\alpha-\frac{k_{1}}{k_{2}}\right| \geq c_{0}^{-1 / 2} \cdot 2^{-1} \cdot\left(k_{2}^{-2}\right)^{\left(\tau_{0}+2\right) / 2}=2^{-1} c_{0}^{-1} \cdot k_{2}^{-\left(\tau_{0}+2\right)}, \text { para } k_{2}^{2} \geq 1
$$

Portanto:

$$
\exists c=2^{-1} c_{0}^{-1}>0 \text { e } \tau=\tau_{0}+2>0 \text { t.q. }\left|\alpha-\frac{k_{1}}{k_{2}}\right| \geq c k_{2}^{-\tau}, \text { se }\left|k_{2}\right| \geq 1,
$$

ou seja, $\alpha$ não é Liouville.

Reciprocamente, se $\alpha$ não é Liouville, então existem $c_{1}, c_{2}>0$ e $\tau>0$ tais que:

$$
\left|\alpha-\frac{k_{1}}{k_{2}}\right| \geq c_{1}\left|k_{2}\right|^{-\tau}, \text { para }\left|k_{2}\right| \geq c_{2}
$$

(podemos supor $c_{2} \geq 1$ ).

Mas

$$
\begin{gathered}
\left|\alpha-\frac{k_{1}}{k_{2}}\right| \geq c_{1}\left|k_{2}\right|^{-\tau}=c_{1}\left(k_{2}^{2}\right)^{-\tau / 2} \geq c_{1}\left(1+k_{2}^{2}\right)^{-\tau / 2} \Rightarrow \\
\left|\alpha-\frac{k_{1}}{k_{2}}\right| \geq c_{1} \frac{\left(1+k_{2}^{2}\right)^{-\tau / 2}}{k_{2}^{2}}, \text { para } k_{2}^{2} \geq 1 .
\end{gathered}
$$

Seja $A=\left\{k_{2} \in \mathbf{Z} \backslash\{0\} ;\left|k_{2}\right|<c_{2}\right\}=\left\{k_{2}^{(1)}, \ldots, k_{2}^{(s)}\right\}$.

Tomemos $\tau_{0}=\tau / 2 \mathrm{e}$

$$
c_{0}^{-1}=\min \left\{c_{1} ;\left|\alpha-\frac{k_{1}}{k_{2}}\right| k_{2}^{2}\left(1+k_{2}^{2}\right)^{\tau / 2} \mid k_{2} \in A, k_{1} \in Z \backslash\{0\}\right\}>0,
$$

pois $0 \notin A$ e $\alpha \in \mathbf{R} \backslash \mathbf{Q}$.

Assim,

$$
\left|\alpha-\frac{k_{1}}{k_{2}}\right| \geq c_{0}^{-1} \frac{\left(1+k_{2}^{2}\right)^{-\tau_{0}}}{k_{2}^{2}}, \text { para todo }\left(k_{1}, k_{2}\right) \in \mathbf{Z}^{2} \backslash\{0\} .
$$

Com base nestes resultados e no fato de que $\alpha=\sqrt{p}, \mathrm{p}$ primo, é um número irracional não-Liouville ${ }^{2}$, podemos observar os seguintes operadores:

\footnotetext{
${ }^{2}$ Referências: [Berg], [Zan]
} 


\subsubsection{Exemplo 1}

Seja $\alpha=\sqrt{p}$, com $p$ primo .

Então o operador: $P=\left(D_{1}-\alpha D_{2}\right)^{2} \dot{\mathrm{e}}(\mathrm{GH})$ em $T^{2}$ e, além disso, $P: C_{0}^{\infty}\left(T^{2}\right) \rightarrow C_{0}^{\infty}\left(T^{2}\right)$ é uma bijeção.

\subsubsection{Exemplo 2}

Seja $\alpha=\sum_{j=1}^{\infty} 10^{-j !}$, o qual é um número de Liouville. Então o operador $(\partial x-\alpha \partial y)^{2}$ não é $(\mathrm{GH}) \mathrm{e}$, portanto, não é uma bijeção em $C_{0}^{\infty}\left(T^{2}\right) .^{3}$

\subsubsection{Exemplo 3}

Seja

$L: C_{0}^{\infty}\left(T^{3}\right) \rightarrow C_{0}^{\infty}\left(T^{3}\right)(n=2)$

$L=\tilde{D}_{1}^{2}+\tilde{D}_{2}^{2}=\left(\partial x_{1}-\alpha_{1} \partial x_{3}\right)^{2}+\left(\partial x_{2}-\alpha_{2} \partial x_{3}\right)^{2}$, com $\alpha_{1}, \alpha_{2} \in \mathbf{R} \backslash \mathbf{Q}$ e nãoLiouville.

Mostraremos que $\alpha_{1}$ e $\alpha_{2}$ satișfazem à condição Diofantina em (2.3) e assim concluímos que $L$ é uma bijeção em $C_{0}^{\infty}\left(T^{3}\right)$. Do corolário (2.2), concluímos que $L$ é $(\mathrm{GH})$ em $T^{3}$ e, além disso, podemos ver que $L(k) \neq 0, \forall k \in \mathbf{Z}^{3} \backslash\{0\}$.

Com efeito, se $\alpha_{1}, \alpha_{2}$ são irracionais não-Liouville, então, pela observação (2.1), existem $c_{0}, \tau_{0}>0$ e $c_{1}, \tau_{1}>0$ tais que:

$$
\left(k_{1}-\alpha_{1} k_{3}\right)^{2} \geq c_{0}^{-1}\left(1+k_{3}^{2}\right)^{-\tau_{0}}, \forall\left(k_{1}, k_{3}\right) \in \mathbf{Z}^{2} \backslash\{0\}
$$

e

$$
\left(k_{2}-\alpha_{2} k_{3}\right)^{2} \geq c_{1}^{-1}\left(1+k_{3}^{2}\right)^{-\tau_{1}}, \forall\left(k_{2}, k_{3}\right) \in \mathbf{Z}^{2} \backslash\{0\}
$$

Logo:

$$
\sum_{j=1}^{2}\left(k_{j}-\alpha_{j} k_{3}\right)^{2} \geq c_{0}^{-1}\left(1+k_{3}^{2}\right)^{-\tau_{0}}+c_{1}^{-1}\left(1+k_{3}^{2}\right)^{-\tau_{1}}, \forall\left(k_{1}, k_{2}, k_{3}\right) \in \mathbf{Z}^{3} \backslash\{0\}
$$

Tomemos $\tau=\max \left\{\tau_{0}, \tau_{1}\right\}$. Dai:

\footnotetext{
${ }^{3}$ Referência: [Berg]
} 


$$
\begin{gathered}
\sum_{j=1}^{2}\left(k_{j}-\dot{\alpha}_{j} k_{3}\right)^{2} \geq\left(c_{0}^{-1}+c_{1}^{-1}\right)\left(1+k_{3}^{2}\right)^{-\tau} \geq c^{-1}\left(1+k_{3}^{2}\right)^{-\tau}, \forall k \in \mathbf{Z}^{3} \backslash\{0\} \\
\operatorname{com} c^{-1}=2 \min \left\{c_{0}^{-1}, c_{1}^{-1}\right\}
\end{gathered}
$$

\subsubsection{Exemplo 4}

Como foi visto no exemplo anterior, podemos generalizar o operador $L$ para dimensões mais altas:

Seja $L: C_{0}^{\infty}\left(T^{n+1}\right) \rightarrow C_{0}^{\infty}\left(T^{n+1}\right)$, dado por:

$$
L=\sum_{j=1}^{n}\left(\partial x_{j}-\alpha_{j} \partial x_{n+1}\right)^{2}
$$

com $\alpha_{j}$ irracional não-Liouville para todo $j=1, \ldots, n$. Então, $L$ é bijeção, é (GH) em $T^{n+1}$ e $L(k) \neq 0, \forall k \in Z^{n+1} \backslash\{0\}$.

Observação 2.2 Se $L=\sum_{j=1}^{n}\left(\partial x_{j}-\alpha_{j} \partial x_{n+1}\right)^{2}$ em $C_{0}^{\infty}\left(T^{n+1}\right)$ tiver algum $\alpha_{i_{0}}=p / q \in \mathbf{Q}, L$ pode deixar de ser injetor. Por exemplo, se $\alpha=p / q \in \mathbf{Q} e$ $L=\sum_{j=1}^{n}\left(\partial x_{j}-\alpha \partial x_{n+1}\right)^{2}$, existem infinitos $k \in \mathbf{Z}^{n+1} \backslash\{0\}$ tais que $e_{k} \in \operatorname{KerL}$.

De fato: se $u \in \operatorname{Ker} L$ e $\hat{u}(k) \neq 0$ para algum $k \in \mathbf{Z}^{n+1} \backslash\{0\}$, então :

$$
\begin{aligned}
\sum_{j=1}^{n}\left(k_{j}-\alpha k_{n+1}\right)^{2} & =0 \Leftrightarrow k_{j}-\alpha k_{n+1}=0, j=1, \ldots, n \\
& \Rightarrow k_{j}-\frac{p}{q} k_{n+1}=0
\end{aligned}
$$

Logo, se $k \in \mathbf{Z}^{n+1}$ e é da forma $k=(l p, \ldots, l p, l q)$, com $l \in \mathbf{Z}$, então $e_{k} \in K e r L$, pois:

$$
\begin{aligned}
L e_{k}(x) & =\sum_{j=1}^{n}\left(\partial x_{j}-\frac{p}{q} \partial x_{n+1}\right)^{2} \cdot e^{i \cdot\left(l p x_{1}+\cdots+l p x_{n}+l q x_{n+1}\right)}= \\
= & i \sum_{j=1}^{n}\left(l p-\frac{p}{q} l q\right)^{2} e^{i .\left(l p x_{1}+\cdots+l p x_{n}+l q x_{n+1}\right)}=0
\end{aligned}
$$


Este operador nos fornece um caso em que $\operatorname{dim} \operatorname{Ker} L$ não é finita e podemos ver que a ele não se aplica o teorema (2.2), uma vez que

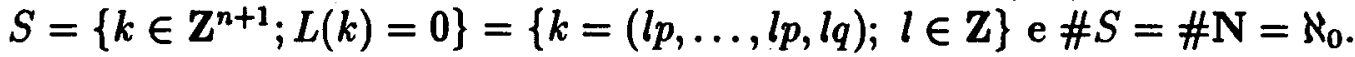

Um outro exemplo em que o operador $P$ tem $\operatorname{dim} \operatorname{Ker} P=\infty$, e que não é $(\mathrm{GH})$ em $T^{2}$, é dado a seguir, mas veremos que $P$ pode ser resolúvel, i.e., $P: \mathcal{D}^{\prime}\left(T^{2}\right) \rightarrow \mathcal{D}^{\prime}\left(T^{2}\right)$ pode ser sobrejetor, se restringirmos o contra-domínio a um subespaço de $C^{\infty}\left(T^{2}\right)$ :

\subsubsection{Exemplo 5}

Seja $P=D_{1}=-i \frac{\partial}{\partial x_{1}}$ definido em $\mathcal{D}^{\prime}\left(T^{2}\right)$. É claro que $P=D_{1}-0 D_{2}$ não é $(\mathrm{GH})$.

Temos $S=\left\{\left(k_{1}, k_{2}\right) \in \mathbf{Z}^{2} ; P\left(k_{1}, k_{2}\right)=0\right\}=\left\{\left(0, k_{2}\right) ; k_{2} \in \mathbf{Z}\right\} \mathrm{e}$ $\operatorname{Ker} P=\left[e_{j} ; j=\left(0, k_{2}\right) \in S\right]$. Logo $\operatorname{dim} \operatorname{Ker} P=\infty$. Consideremos

$$
C_{S}^{\infty}\left(T^{2}\right)=\left\{f \in C^{\infty}\left(T^{2}\right) ; \hat{f}(0, m)=0, \forall m \in \mathbf{Z}\right\}=\left\{f-\pi_{S} f ; f \in C^{\infty}\left(T^{2}\right)\right\} .
$$

Para que, dada $f \in C^{\infty}\left(T^{2}\right)$, exista $u \in \mathcal{D}^{\prime}\left(T^{2}\right)$ tal que $P u=f$, devemos ter:

$$
\begin{aligned}
D_{1} u & =-i \frac{\partial u}{\partial x_{1}}=f \Rightarrow \\
u\left(x_{1}, x_{2}\right) & =i \int_{0}^{x_{1}} f\left(t, x_{2}\right) d t+g\left(x_{2}\right),
\end{aligned}
$$

onde $\mathrm{g}$ deve ser $2 \pi$-periódica.

Para que $u$ seja $2 \pi$-periódica em cada variável, é necessário e suficiente que:

$$
u\left(x_{1}+2 \pi, x_{2}\right)=u\left(x_{1}, x_{2}\right)=u\left(x_{1}, x_{2}+2 \pi\right), \forall\left(x_{1}, x_{2}\right) \in \mathbf{R}^{2} .
$$

No caso em que $u$ é solução da equação acima temos:

$$
\begin{aligned}
u\left(x_{1}, x_{2}+2 \pi\right) & =i \int_{0}^{x_{1}} f\left(t, x_{2}+2 \pi\right) d t+g\left(x_{2}+2 \pi\right) \\
& =\int_{0}^{x_{1}} f\left(t, x_{2}\right) d t+g\left(x_{2}\right)=u\left(x_{1}, x_{2}\right), \forall x_{1}, x_{2} .
\end{aligned}
$$


Ainda:

$$
\begin{gathered}
u\left(x_{1}+2 \pi, x_{2}\right)=u\left(x_{1}, x_{2}\right) \Leftrightarrow \\
\int_{0}^{x_{1}+2 \pi} f\left(t, x_{2}\right) d t=\int_{0}^{x_{1}} f\left(t, x_{2}\right) d t \Leftrightarrow \\
\int_{0}^{x_{1}} f\left(t, x_{2}\right) d t+\int_{x_{1}}^{x_{1}+2 \pi} f\left(t, x_{2}\right) d t=\int_{0}^{x_{1}} f\left(t, x_{2}\right) d t \Leftrightarrow \\
\int_{x_{1}}^{x_{1}+2 \pi} f\left(t, x_{2}\right) d t=0
\end{gathered}
$$

Mas:

$$
\begin{aligned}
& \int_{x_{1}}^{x_{1}+2 \pi} f\left(t, x_{2}\right) d t \stackrel{0<x_{1}<2 \pi}{=} \int_{x_{1}}^{2 \pi} f\left(t, x_{2}\right) d t+\int_{2 \pi}^{2 \pi+x_{1}} f\left(t, x_{2}\right) d t \stackrel{y=t-2 \pi}{=} \\
&=\int_{x_{1}}^{2 \pi} f\left(t, x_{2}\right) d t+\int_{0}^{x_{1}} f\left(y+2 \pi, x_{2}\right) d y \\
&=\int_{x_{1}}^{2 \pi} f\left(t, x_{2}\right) d t+\int_{0}^{x_{1}} f\left(y, x_{2}\right) d y=\int_{0}^{2 \pi} f\left(t, x_{2}\right) d t
\end{aligned}
$$

Portanto,

$$
u\left(x_{1}+2 \pi, x_{2}\right)=u\left(x_{1}, x_{2}\right) \Leftrightarrow \int_{0}^{2 \pi} f\left(t, x_{2}\right) d t=0
$$

E temos:

$$
\begin{aligned}
0 & =\int_{0}^{2 \pi} f\left(t, x_{2}\right) d t=c \int_{0}^{2 \pi} e^{i m x_{2}} d x_{2} \cdot\left[\int_{0}^{2 \pi} e^{i .0 \cdot x_{1}} f\left(x_{1}, x_{2}\right) d x_{1}\right] \\
& =c \cdot \int_{0}^{2 \pi} \int_{0}^{2 \pi} e^{i\left(0 x_{1}+m x_{2}\right)} f\left(x_{1}, x_{2}\right) d x_{1} d x_{2}=c \hat{f}(0, m), \forall m \in \mathbf{Z} .
\end{aligned}
$$

Portanto $u \in \mathcal{D}^{\prime}\left(T^{2}\right) \Leftrightarrow f \in C_{S}^{\infty}\left(T^{2}\right)$.

Porém, mesmo que $f \in C_{S}^{\infty}$, não podemos garantir que $u \in C_{S}^{\infty}$, uma vez que o operador não é (GH). Por exemplo, $f \equiv 0 \in C_{S}^{\infty}\left(T^{2}\right)$;

$$
u\left(x_{1}, x_{2}\right)=\left|\cos x_{2}\right|^{3} \in \mathcal{D}^{\prime}\left(T^{2}\right) \backslash C^{\infty}\left(T^{2}\right) \text { e } P u=D_{1}\left(\left|\cos x_{2}\right|^{3}\right)=0 .
$$

O exemplo que segue fornece um operador que não é (GH) em $T^{2}$ e não é resolúvel em $\mathcal{D}^{\prime}\left(T^{2}\right)$. 


\subsubsection{Exemplo 6}

Seja $P=D_{1}-\alpha D_{2}$, com $\alpha$ irracional Liouville.

Definimos $S=\left\{\left(k_{1}, k_{2}\right) ; P\left(k_{1}, k_{2}\right)=0\right\}=\{(0,0)\}$ e $\operatorname{Ker} P=\{c ; c \in \mathbf{R}\}=\left[e_{0}=1\right]$.

Como já foi visto, $P$ não é $(\mathrm{GH})$.

Afirmação : é possível construir $f \in C^{\infty}\left(T^{2}\right)$ de modo que não exista $u \in \mathcal{D}^{\prime}\left(T^{2}\right)$ tal que $P u=f$.

De fato: como $\alpha$ é Liouville, existe uma sequência $\left(m_{j}, n_{j}\right) \in \mathbf{Z}^{2}$ tal que:

$$
\left(m_{j}, n_{j}\right) \rightarrow \infty \text { quando } j \rightarrow \infty \text { e }\left|P\left(m_{j}, n_{j}\right)\right| \leq \frac{1}{\left(m_{j}^{2}+n_{j}^{2}\right)^{j}} .
$$

Tomemos

$$
f(x, y)=\sum_{j \in N} \frac{1}{\left(m_{j}^{2}+n_{j}^{2}\right)^{j / 2}} e^{i\left(m_{j} x+n_{j} y\right)}
$$

e assim teremos:

$$
\begin{cases}\hat{f}(m, n)=0 & \text { se }(m, n) \neq\left(m_{j}, n_{j}\right) \text { para todo } j \in \mathbf{N} \\ \hat{f}\left(m_{j}, n_{j}\right)=\frac{1}{\left(n_{j}^{2}+m_{j}^{2}\right)^{j / 2}} & j \in \mathbf{N}\end{cases}
$$

- $f \in C^{\infty}\left(T^{2}\right)$, pois:

$$
\begin{aligned}
& \sup _{(m, n)}\left\{\frac{|\hat{f}(m, n)|}{\left(n^{2}+m^{2}+1\right)^{k}}\right\}=\sup _{j \in \mathbb{N}}\left\{\frac{\left|\hat{f}\left(m_{j}, n_{j}\right)\right|}{\left(n_{j}^{2}+m_{j}^{2}+1\right)^{k}}\right\}= \\
& =\sup _{j \in \mathbb{N}}\left\{\frac{1}{\left(n_{j}^{2}+m_{j}^{2}+1\right)^{k}\left(n_{j}^{2}+m_{j}^{2}\right)^{j / 2}}\right\}<\infty, \forall k \in \mathbf{Z} .
\end{aligned}
$$

Suponhamos que exista $u \in \mathcal{D}^{\prime}\left(T^{2}\right)$ tal que $P u=f$. Então:

$$
P(m, n) \hat{u}(m, n)=\hat{f}(m, n), \forall(m, n) \in \mathbf{Z}^{2} .
$$


Em particular:

$$
\begin{aligned}
\hat{u}\left(m_{j}, n_{j}\right) \mid & =\frac{\left|\hat{f}\left(m_{j}, n_{j}\right)\right|}{\left|P\left(m_{j}, n_{j}\right)\right|} \\
& \geq \frac{\left(n_{j}^{2}+m_{j}^{2}\right)^{j}}{\left(n_{j}^{2}+m_{j}^{2}\right)^{j / 2}}=\left(n_{j}^{2}+m_{j}^{2}\right)^{j / 2} \rightarrow \infty \text { quando } j \rightarrow \infty .
\end{aligned}
$$

Vemos, então, que $\left(\hat{u}\left(m_{j}, n_{j}\right)\right)_{j \in N}$ não tem crescimento lento (Absurdo!). 


\section{Capítulo 3}

\section{Hipoeliticidade Global para uma Classe de Operadores em $T^{n}$ com Coeficientes Variáveis}

Neste capítulo serão vistos resultados de [Yos] sobre hipoeliticidade global para operadores em $T^{n}, n \geq 2$, da forma:

$$
P=p\left(D_{1}^{2}\right)+e^{i x_{1}}+a e^{-i x_{1}}
$$

onde $p(t)$ é um polinômio a valores complexos de ordem $m \geq 1, t \in \mathbf{R} e a= \pm 1$, o qual deverá satisfazer a certas hipóteses. Observamos que este tipo de operador tem a parte principal (derivadas de maior ordem) a coeficientes constantes e o único coeficiente variável é o termo de ordem zero. Ressaltamos, ainda, que a hipoeliticidade global de um operador mais geral também foi abordada em [Yos], embora sob diferentes hipóteses; porém, seu estudo detalhado não será incluído neste trabalho. Este resultado será mencionado na seção (3.5) (teorema 3.3) e foi deixado como objeto de estudos futuros.

O teorema central será abordado nas seções (3.1) e (3.3), sendo demonstrado nesta última. forma:

A seção (3.2) tratará de resultados relativos a uma equação de diferenças da

$$
v_{j+2}=a_{j} v_{j+1}+v_{j}-f_{j}, \quad j=0, \pm 1, \pm 2, \ldots,
$$

onde $a_{j}, f_{j} \in \mathbf{C}$. Esta equação surgirá ao longo de várias demonstrações. 
Na seção (3.4) serão feitas algumas observações relevantes acerca do teorema principal e sua prova.

Um outro resultado, cuja demonstração seguirá os moldes da seção (3.3), será visto na seção (3.5), agora para um operador da forma:

$$
Q=q\left(D^{\prime}\right)+2 \cos x_{1}
$$

onde :

$$
q\left(D^{\prime}\right)=\sum_{\substack{\alpha \in \mathbb{Z}^{n-1} \\|a| \leq m}} c_{\alpha} D_{2}^{\alpha_{2}} \ldots D_{n}^{\alpha_{n}} \text { e } D_{j}=\frac{1}{i} \frac{\partial}{\partial x_{j}}, j=2, \ldots, n
$$

\subsection{Hipoeliticidade Global para um Operador da forma: $P=p\left(D_{1}^{2}\right)+e^{i x_{1}}+a e^{-i x_{1}}$}

Seja $p(t)$ um polinômio de ordem $m \geq 1$, com $t \in \mathbf{R}$ e $a= \pm 1$. Consideremos a equação:

$$
P u=\left(p\left(D_{1}^{2}\right)+e^{i x_{1}}+a e^{-i x_{1}}\right) u(x)=f(x) e m T^{n}, n \geq 2
$$

Suponhamos que $p$ satisfaça as seguintes condições:

$$
\text { (A.1) } \quad p(0)=0 ;|p(1)| \geq 1 ;\left|p\left(t^{2}\right)\right|>2 \text { se } t \geq 2, t \in \mathbf{N} \text {. }
$$

É fácil ver que $P$ não é elítico, pois:

$$
P_{m}(k)=p_{m}\left(k_{1}^{2}\right)=0, \text { para todo } k=\left(0, k_{2}, \ldots, k_{n}\right) \in \mathbf{Z}^{n} .
$$

Afirmação: $P$ também não é hipoelítico.

De fato, existe um aberto $\Omega \subset T^{1}$ e existe $v\left(x_{1}\right) \not \equiv 0 \in C^{\infty}(\Omega)$ tal que $P v=0$.

Com efeito, se procurarmos $v\left(x_{1}\right)$ tal que $\left(p\left(D_{1}^{2}\right)+e^{i x_{1}}+a e^{-i x_{1}}\right) v\left(x_{1}\right)=0$, com as condiçốes iniciais: $v(0)=0 ; v^{\prime}(0)=0 ; \ldots, v^{(2 m-1)}(0)=1$, teremos, agora, um problema de equações diferenciais ordinárias, o qual sabemos admitir solução 
$v\left(x_{1}\right) \in C^{2 m}$ em uma vizinhança $(\Omega)$ da origem, pois todos os coeficientes são $C^{\infty}$ (ver (1.5.3)). Observando o símbolo deste operador agindo em uma única variável, $x_{1}$, temos:

$$
P_{m}\left(k_{1}\right)=p_{m}\left(k_{1}^{2}\right)=0 \Leftrightarrow k_{1}=0 .
$$

Logo, ele é elítico quando agindo somente em $x_{1}$ e, portanto, $v\left(x_{1}\right) \in C^{\infty}(\Omega)$.

Tomando $w\left(x^{\prime}\right)=w\left(x_{2}, \ldots, x_{n}\right) \in \mathcal{D}^{\prime}\left(T^{n}\right) \backslash C^{\infty}\left(T^{n}\right)$ e $u(x)=v\left(x_{1}\right) w\left(x^{\prime}\right)$ teremos:

$$
\begin{gathered}
P u=p\left(D_{1}^{2}\right) v\left(x_{1}\right) w\left(x^{\prime}\right)+\left[e^{i x_{1}}+a e^{-i x_{1}}\right] v\left(x_{1}\right) w\left(x^{\prime}\right)= \\
=w\left(x^{\prime}\right) \cdot\left[\left(p\left(D_{1}^{2}\right)+e^{i x_{1}}+a e^{-i x_{1}}\right) v\left(x_{1}\right)\right]=w\left(x^{\prime}\right) \cdot 0=0
\end{gathered}
$$

Portanto, $u$ é solução para a equação $P u=0$, mas $u \notin C^{\infty}$, ou seja, $P$ não é (H).

Entretanto, o operador $P$ é (GH), como mostra o resultado a seguir:

Teorema 3.1 Seja $P=p\left(D_{1}^{2}\right)+e^{i x_{1}}+a e^{-i x_{1}}$ e suponhamos que as condições (A.1) sejam válidas. Entâa, $P$ é $(G H)$ em $T^{n}$.

Antes de demostrarmos o teorema, analisemos alguns exemplos:

\subsubsection{Exemplo 1}

Se $a=1$ e $p(t)=t$, o operador $P$ fica:

$$
P=D_{1}^{2}+2 \cos x_{1},
$$

o qual é (GH) em $T^{2}$, pois $p(t)$ satisfaz as condições $(A .1)$. Ainda, se $0<r \leq 1$, consideremos o operador:

$$
P=D_{1}^{2}+2 r \cos x_{1} \text {. }
$$

Neste caso, ainda podemos aplicar o teorema anterior, uma vez que:

$$
P=r\left(\frac{D_{1}^{2}}{r}+2 \cos x_{1}\right) \text { e } \tilde{p}(t)=\frac{t}{r}
$$

ainda satisfaz as hipóteses em $(A .1)$ :

$$
\tilde{p}(0)=0 ;|\tilde{p}(1)|=\frac{1}{r} \geq 1 ;\left|\tilde{p}\left(t^{2}\right)\right|=\frac{t^{2}}{r} \geq t^{2}>2, \text { se } t \in N, t \geq 2 \text {. }
$$




\subsubsection{Exemplo 2}

Se $a=-1, p(t)=t$, o operador $P$ fica:

$$
P=D_{1}^{2}+2 i \operatorname{sen} x_{1}
$$

e é (GH) em $T^{2}$.

Como foi visto no exemplo anterior, o teorema também se aplica ao operador

$$
P=D_{1}^{2}+2 \text { risen } x_{1}, \text { quando } 0<r \leq 1 .
$$

Os casos dos exemplos anteriores, $\operatorname{com} r>1$, serão analisados posteriormente.

\subsubsection{Exemplo 3}

O polinômio $p$ pode apresentar coeficientes complexos, desde que satisfaça as condições $(A .1)$ :

Se $p(t)=t^{2}+4 i t$, temos: $p(0)=0 ;|p(1)|=\sqrt{1+4^{2}} \geq 1 \mathrm{e}$

$$
\left|p\left(t^{2}\right)\right|=\sqrt{t^{4}+16 t^{2}}=|t| \sqrt{16+t^{2}}>2 \text {, se } t \geq 2, t \in \mathrm{N} .
$$

Logo, o operador $P=D_{1}^{4}+4 i D_{1}^{2}+e^{i x_{1}}+a e^{-i x_{1}}$, com $a= \pm 1$, é (GH) em $T^{n}, n \geq 2$.

Assim, conseguimos uma classe significante de operadores de ordem maior ou igual a 2 que é (GH), enquanto que no capítulo anterior, os exemplos mais facilmente encontrados tinham apenas ordem 2 , em sua maioria.

\subsection{Alguns Resultados sobre uma Equação de Diferenças}

$\mathrm{Na}$ demonstração do teorema (3.1) serão utilizados alguns resultados envolvendo a resolução de uma equação de diferenças da forma:

$$
v_{j+2}=a_{j} v_{j+1}+v_{j}-f_{j}, j=0, \pm 1, \pm 2, \ldots, \text { com } a_{j}, f_{j} \in \mathbf{C} \text { para todo } j \in \mathbf{Z} .
$$


Nesta seção trataremos destes resultados:

Lema 3.1 Definamos, para $j=1,2, \ldots$.

$$
\left\{\begin{array}{lll}
p_{j+2}=a_{j}(\sigma) p_{j+1}+p_{j} ; & p_{1}=1 ; \quad p_{2}=0 \\
q_{j+2}=a_{j}(\sigma) q_{j+1}+q_{j} ; & q_{1}=0 ; \quad q_{2}=1
\end{array} e\right.
$$

onde $a_{j}(\sigma) \in \mathbf{C}$ e depende de um parâmetro $\sigma \in \mathbf{Z}^{n-1}$.

Então, $\forall j=1,2, \ldots e \forall \sigma \in \mathbf{Z}^{n-1}$, vale:

$$
p_{j+1} q_{j}-p_{j} q_{j+1}=(-1)^{j}
$$

Prova: Usaremos indução finita:

- para $j=1$, temos: $p_{2} q_{1}-q_{2} p_{1}=0-1=-1$.

- suponhamos que a equação anterior seja válida para $j=s \in \mathbf{N}$ e mostremos que também é válida para $j=s+1$ :

$$
\begin{aligned}
p_{s+2} q_{s+1}-p_{s+1} q_{s+2} & =\left(a_{s}(\sigma) p_{s+1}+p_{s}\right) q_{s+1}-p_{s+1}\left(a_{s}(\sigma) q_{s+1}+q_{s}\right) \\
& =a_{s}(\sigma) p_{s+1} q_{s+1}+p_{s} q_{s+1}-a_{s}(\sigma) p_{s+1} q_{s+1}-p_{s+1} q_{s} \\
& =-\left(p_{s+1} q_{s}-p_{s} q_{s+1}\right)=-(-1)^{s}=(-1)^{s+1}
\end{aligned}
$$

Lema 3.2 Sejam $p_{j}, q_{j}$ definidos como no lema (3.1) e suponha que exista $K>1$, independente de $j$ e $\sigma$, tal que:

$$
\left|a_{1}(\sigma)\right| \geq 1 \text { e }\left|a_{j}(\sigma)\right| \geq 1+K \text { para } j \geq 2 \text { e para todo } \sigma \in \mathbf{Z}^{n} .
$$

Então, para todo $j \geq 4$, vale:

$$
\left|q_{j}\right| \geq K^{j-3} ; \quad\left|p_{j}\right| \geq K^{j-3}
$$


Prova: Da definição de $p_{j}$ e $q_{j}$, como $\left|a_{1}\right| \geq 1$ e $\left|a_{j}\right| \geq 1+K, j \geq 2$, temos:

$$
\begin{gathered}
\left|q_{3}\right|=\left|a_{1} q_{2}+q_{1}\right|=\left|a_{1}\right| \geq 1 . \\
\left|q_{4}\right|=\left|a_{2} q_{3}+q_{2}\right|=\left|q_{3}\right|\left|a_{2}+\frac{q_{2}}{q_{3}}\right| \geq\left|q_{3}\right|\left(\left|a_{2}\right|-1\right) \geq K .
\end{gathered}
$$

Suponha, agora, que $\left|q_{j}\right| \geq K^{j-3}$ para todo $4 \leq j \leq N$. Então:

$$
\begin{aligned}
\left|q_{N+1}\right| & =\left|a_{N-1} q_{N}+q_{N-1}\right| \geq\left|q_{N}\right|\left|a_{N-1}\right|-\frac{\left|q_{N-1}\right|}{\left|q_{N}\right|} \mid \geq \\
& \geq K^{N-3}\left(\left|a_{N-1}\right|-1\right) \geq K^{N-2} .
\end{aligned}
$$

A desigualdade para os $p_{j}^{\prime} s$ segue de maneira análoga.

Observação 3.1 Uma desigualdade válida que será bastante utilizada e que segue da demostração anterior é: se $j \geq 4$ e $r \in \mathrm{N}$ :

$$
\left|q_{j+r}\right|=\left|a_{j+r-2} q_{j+r-1}+q_{j+r-2}\right| \geq\left|q_{j+r-1}\right|\left(\left|a_{j+r-2}\right|-1\right) \geq\left|q_{j+r-1}\right| . K .
$$

Do mesmo modo, podemos ver que: $\left|q_{j+r-1}\right| \geq K .\left|q_{j+r-2}\right|$ e assim sucessivamente. Logo:

$$
\left|q_{j+r}\right| \geq K^{r}\left|q_{j}\right|
$$

E o mesmo vale para $p_{j}$ :

$$
\left|p_{j+r}\right| \geq K^{r}\left|p_{j}\right|
$$

Lema 3.3 Definimos:

$$
t=\sum_{j=2}^{\infty} \frac{(-1)^{j}}{q_{j} q_{j+1}},
$$

onde $q_{j}$ é definido como no lema (3.1). Então, sob as hipóteses do lema (3.2), existem $K_{0}>0$ e $j_{0} \geq 1$, independentes de $j$ e $\sigma$, tais que:

$$
\left|p_{j}-t q_{j}\right| \leq K_{0}\left|q_{j}\right|^{-1}, \quad \forall j \geq j_{0} .
$$


Prova: $t$ está bem definido, uma vez que, para $j \geq 4$, temos :

$$
\frac{1}{\left|q_{j}\right|\left|q_{j+1}\right|} \leq \frac{1}{K^{j-3} K^{j-2}}=\frac{1}{K^{2 j-5}}
$$

e $2 j-5 \geq 2, \forall \cdot j \geq 4$ e $K>1$.

É fácil ver que, para $p_{j}$ e $q_{j}$ definidos como no lema (3.1), vale:

$$
\frac{p_{j+1}}{q_{j+1}}=\sum_{k=2}^{j} \frac{(-1)^{k}}{q_{k} q_{k+1}}+\frac{p_{2}}{q_{2}}=\sum_{k=2}^{j} \frac{(-1)^{k}}{q_{k} q_{k+1}} \text { pois } p_{2}=0
$$

Para isto, foi usada, recursivamente, a igualdade:

$$
\frac{p_{j+1}}{q_{j+1}}-\frac{p_{j}}{q_{j}}=\frac{(-1)^{j}}{q_{j} q_{j+1}} .
$$

E temos:

$$
\begin{aligned}
\left|t-\frac{p_{j}}{q_{j}}\right| & \leq \sum_{k=j}^{\infty} \frac{1}{\left|q_{k}\right|\left|q_{k+1}\right|} \\
& =\frac{1}{\left|q_{j}\right|^{2}}\left[\frac{\left|q_{j}\right|^{2}}{\left|q_{j} q_{j+1}\right|}+\frac{\left|q_{j}\right|^{2}}{\left|q_{j+1} q_{j+2}\right|}+\cdots\right]
\end{aligned}
$$

e para $j \geq j_{0}=4$, vale a desigualdade da observação (3.1) e assim:

$$
\frac{\left|q_{j}\right|^{2}}{\left|q_{j+r} q_{j+r+1}\right|} \leq \frac{\left|q_{j}\right|^{2}}{K^{r} K^{r+1}\left|q_{j}\right|^{2}}=\frac{1}{K^{2 r+1}}
$$

e portanto:

$$
\sum_{r=0}^{\infty} \frac{\left|q_{j}\right|^{2}}{\left|q_{j+r} q_{j+r+1}\right|} \leq \frac{1}{K} \sum_{r=0}^{\infty} \frac{1}{K^{2 r}}=K_{0}<\infty, \text { pois } K>1
$$

Logo:

$$
\left|p_{j}-t q_{j}\right|=\left|q_{j}\right|\left|t-\frac{p_{j}}{q_{j}}\right| \leq\left|q_{j}\right| \frac{K_{0}}{\left|q_{j}\right|^{2}}=K_{0}\left|q_{j}\right|^{-1}, j \geq j_{0} .
$$


Observação $3.2 t$ definido no lema (3.3) é não-nulo.

Com efeito, se $t$ fosse nulo, teríamos: para $j \geq j_{0} \geq 4$,

$$
\begin{gathered}
K^{j-3} \leq\left|p_{j}\right| \leq K_{0}\left|q_{j}\right|^{-1} \leq \frac{K_{0}}{K^{j-3}} \Rightarrow \\
\Rightarrow K_{0} \geq K^{2(j-3)} \rightarrow \infty \text { quando } j \rightarrow \infty \text { (Absurdo!). }
\end{gathered}
$$

Lema 3.4 Consideremos a equação de diferenças:

$$
v_{j+2}=a_{j}(\sigma) v_{j+1}+v_{j}-f_{j}, j=1,2, \ldots,
$$

com valores iniciais $v_{1}=\alpha ; v_{2}=\beta$. Então, uma solução para (3.1) é:

$(*)\left\{\begin{array}{l}v_{j}=\alpha p_{j}+\beta q_{j}+\varphi_{j}, j=1,2, \ldots \\ \varphi_{1}=\varphi_{2}=0 \text { e se } j \geq 3: \\ \varphi_{j}=\sum_{\nu=1}^{j-3} f_{\nu} \cdot(-1)^{\nu}\left\{p_{j} q_{\nu+1}-q_{j} p_{\nu+1}\right\}-f_{j-2}= \\ =\left(p_{j}-t q_{j}\right) \sum_{\nu 1}^{j-3} f_{\nu \cdot} \cdot(-1)^{\nu} q_{\nu+1}+q_{j}\left\{\gamma_{1}-\sum_{\nu=1}^{j-3} f_{\nu} \cdot(-1)^{\nu}\left(p_{\nu+1}-t q_{\nu+1}\right)\right\}- \\ -f_{j-2}-\gamma_{1} q_{j} \equiv T_{1}+T_{2}-f_{j-2}-\gamma_{1} q_{j}\end{array}\right.$

onde os $p_{j}^{\prime}$ s e $q_{j}^{\prime} s$ são definidos como no lema (3.1) e:

$$
\gamma_{1}=\sum_{\nu=1}^{\infty} f_{\nu} \cdot(-1)^{\nu}\left(p_{\nu+1}-t q_{\nu+1}\right)
$$

Prova: Primeiro, observemos que, na expressão de $\varphi_{j}$, a segunda igualdade foi obtida somando-se e subtraindo-se os termos: $\left(t q_{j} \cdot \sum_{\nu=1}^{j-3} f_{\nu}(-1)^{\nu} q_{\nu+1}\right)$ e $\left(\gamma_{1} q_{j}\right)$; na terceira igualdade, tomamos $T_{1}=\left(p_{j}-t q_{j}\right) \sum_{\nu=1}^{j-3} f_{\nu}(-1)^{\nu} q_{\nu+1}$ e $T_{2}=q_{j}\left\{\gamma_{1}-\sum_{\nu=1}^{j-3} f_{\nu}(-1)^{\nu}\left(p_{\nu+1}-t q_{\nu+1}\right\}\right.$.

É fácil ver que $v_{j}^{\prime}=\alpha p_{j}+\beta q_{j}$ é solução da equação (3.1) se $f_{j}=0, \forall j=1,2, \ldots$

Definimos:

$$
A_{j, \nu}=p_{j} q_{\nu}-p_{\nu} \dot{q}_{j}
$$


Temos que $A_{j, j}=0$ e pelo lema (3.1):

$$
A_{j+1, j}=(-1)^{j} ; A_{j, j-1}=(-1)^{j-1}, \ldots
$$

Ainda se $j=1,2, \ldots, \forall \nu \in \mathbf{N}$, vale:

$$
\begin{aligned}
A_{j+2, \nu} & =p_{j+2} q_{\nu}-p_{\nu} q_{j+2}=\left(a_{j} p_{j+1}+p_{j}\right) q_{\nu}-p_{\nu}\left(a_{j} q_{j+1}+q_{j}\right) \\
& =a_{j}\left(p_{j+1} q_{\nu}-p_{\nu} q_{j+1}\right)+\left(p_{j} q_{\nu}-p_{\nu} q_{j}\right) \\
& =a_{j} A_{j+1, \nu}+A_{j, \nu}
\end{aligned}
$$

Suponhamos, agora, por indução, que $v_{j}=\alpha p_{j}+\beta q_{j}+\varphi_{j}, j=1,2, \ldots$ seja solução da equação (3.1) se $j \leq N$, i.e., suponhamos que tais $v_{j}^{\prime} s$ satisfaçam:

$$
v_{j+2}=a_{j} v_{j+1}+v_{j}-f_{j}, j \leq \mathrm{N}
$$

Mostremos que $v_{\mathrm{N}+1}=\alpha p_{\mathrm{n}+1}+\beta q_{\mathrm{N}+1}+\varphi_{\mathrm{N}+1}$ também satisfaz à equação (3.1):

De fato:

$$
\begin{gathered}
v_{\mathrm{N}+1+2}=v_{\mathrm{N}+3}=\alpha p_{\mathrm{N}+3}+\beta q_{\mathrm{N}+3}+\varphi_{\mathrm{N}+3}= \\
=\alpha\left(a_{\mathrm{N}+1} p_{\mathrm{N}+2}+p_{\mathrm{N}+1}\right)+\beta\left(a_{\mathrm{N}+1} q_{\mathrm{N}+2}+q_{\mathrm{N}+1}\right)+\sum_{\nu=2}^{\mathrm{N}+3-2} f_{\nu-1}(-1)^{\nu-1} A_{\mathrm{N}+3, \nu}-f_{\mathrm{N}+1}= \\
=a_{\mathrm{N}+1}\left(\alpha p_{\mathrm{N}+2}+\beta q_{\mathrm{N}+2}\right)+\left(\alpha p_{\mathrm{N}+1}+\beta q_{\mathrm{N}+1}\right)+ \\
+\sum_{\nu=2}^{\mathrm{N}+1} f_{\nu-1}(-1)^{\nu-1}\left(a_{\mathrm{N}+1} A_{\mathrm{N}+2, \nu}+A_{\mathrm{N}+1, \nu}\right)-f_{\mathrm{N}+1}= \\
=a_{\mathrm{N}+1}\left(\alpha p_{\mathrm{N}+2}+\beta q_{\mathrm{N}+2}+\sum_{\nu=2}^{\mathrm{N}+1} f_{\nu-1}(-1)^{\nu-1} A_{\mathrm{N}+2, \nu}+\right. \\
+\left(\alpha p_{\mathrm{N}+1}+\beta q_{\mathrm{N}+1}+\sum_{\nu=2}^{\mathrm{N}+1} f_{\nu-1}(-1)^{\nu-1} A_{\mathrm{N}+1, \nu}\right)-f_{\mathrm{N}+1}=
\end{gathered}
$$




$$
\begin{aligned}
& =a_{\mathrm{N}+1}\left(\alpha p_{\mathrm{N}+2}+\beta q_{\mathrm{N}+2}+\sum_{\nu=2}^{\mathrm{N}} f_{\nu-1}(-1)^{\nu-1} A_{\mathrm{N}+2, \nu}+f_{\mathrm{N}}(-1)^{\mathrm{N}} A_{\mathrm{N}+2, \mathrm{~N}+1}\right)+ \\
& +\left(\alpha p_{\mathrm{N}+1}+\beta q_{\mathrm{N}+1}+\sum_{\nu=2}^{\mathrm{N}-1} f_{\nu-1}(-1)^{\nu-1} A_{\mathrm{N}+1, \nu}+f_{\mathrm{N}-1}(-1)^{\mathrm{N}-1} A_{\mathrm{N}+1, \mathrm{~N}}+\right. \\
& +f_{\mathrm{N}}(-1)^{\mathrm{N}} A_{\mathrm{N}+1, \mathrm{~N}+1}-f_{\mathrm{N}+1}= \\
& =a_{\mathrm{N}+1}\left(\alpha p_{\mathrm{N}+2}+\beta q_{\mathrm{N}+2}+\sum_{\nu=2}^{\mathrm{N}} f_{\nu-1}(-1)^{\nu-1} A_{\mathrm{N}+2, \nu}-f_{\mathrm{N}}\right)+ \\
& +\left(\alpha p_{\mathrm{N}+1}+\beta q_{\mathrm{N}+1}+\sum_{\nu=2}^{\mathrm{N}-1} f_{\nu-1}(-1)^{\nu-1} A_{\mathrm{N}+1, \nu}-f_{\mathrm{N}-1}\right)-f_{\mathrm{N}+1}= \\
& =a_{N+1}\left(\alpha p_{N+2}+\beta q_{N+2}+\varphi_{N+2}\right)+\left(\alpha p_{N+1}+\beta q_{N+1}+\varphi_{N+1}\right)-f_{N+1}= \\
& =a_{\mathrm{N}+1} v_{\mathrm{N}+2}+v_{\mathrm{N}+1}-f_{\mathrm{N}+1} \text {. }
\end{aligned}
$$

\subsection{Demonstração do teorema central}

Seja $u \in \mathcal{D}^{\prime}\left(T^{n}\right)$ tal que $P u=f \in C^{\infty}\left(T^{n}\right)$. Consideremos as expansões em séries de Fourier:

$$
u(x)=\sum_{k \in \mathbf{Z}^{n}} \hat{u}(k) e^{i k . x} \text { e } f(x)=\sum_{k \in Z^{n}} \hat{f}(k) e^{i k . x},
$$


onde $k=\left(k_{1}, \ldots, k_{n}\right) ; x=\left(x_{1}, \ldots, x_{n}\right)$.

Substituindo-as na equação $P u=f$, teremos:

$$
\sum_{k} p\left(k_{1}^{2}\right) \hat{u}(k) e^{i k \cdot x}+\sum_{k} \hat{u}(k) e^{i\left(k+e_{1}\right) \cdot x}+a \sum_{k} \hat{u}(k) e^{i\left(k-e_{1}\right) \cdot x}=\sum_{k} \hat{f}(k) e^{i k . x},
$$

onde $e_{1}=(1,0, \ldots, 0) \in \mathbf{Z}^{n}$.

Logo:

$$
\sum_{k}\left[p\left(k_{1}^{2}\right) \hat{u}(k)+\hat{u}\left(k-e_{1}\right)+a \hat{u}\left(k+e_{1}\right)\right] \cdot e^{i k . x}=\sum_{k} \hat{f}(k) e^{i k . x}
$$

e para todo $k \in \mathbf{Z}^{n}$ :

$$
p\left(k_{1}^{2}\right) \hat{u}(k)+\hat{u}\left(k-e_{1}\right)+a \hat{u}\left(k+e_{1}\right)=\hat{f}(k),
$$

ou seja,

$$
a \hat{u}\left(k+e_{1}\right)=-p\left(k_{1}^{2}\right) \hat{u}(k)-\hat{u}\left(k-e_{1}\right)+\hat{f}(k)
$$

Fixemos $k^{\prime}=\left(k_{2}, \ldots, k_{n}\right) \in \mathrm{Z}^{n-1}$ e façamos:

$$
k_{1}=j ; v_{j}=(\sqrt{-a})^{j} \hat{u}\left(j-1, k^{\prime}\right) ; a_{j}=\frac{p\left(j^{2}\right)}{\sqrt{-a}} ; f_{j}=\hat{f}\left(j, k^{\prime}\right) \cdot(\sqrt{-a})^{j} .
$$

Então, de (3.2), segue que:

$$
v_{j+2}=a_{j} v_{j+1}+v_{j}-f_{j}, j=0, \pm 1, \ldots
$$

De fato:

- Se $a=-1$, então $(\sqrt{-a})^{j}=1, \forall j$ e teremos:

$$
j=k_{1} ; v_{j}=\hat{u}\left(j-1, k^{\prime}\right) ; a_{j}=p\left(j^{2}\right) ; f_{j}=\hat{f}\left(j, k^{\prime}\right) .
$$

Daí: $v_{j+2}=\hat{u}\left(j+1, k^{\prime}\right) ; v_{j+1}=\hat{u}\left(j, k^{\prime}\right)$ e $(3.2)$ fica:

$$
\begin{aligned}
(-1) \hat{u}\left(j+1, k^{\prime}\right) & =-p\left(j^{2}\right) \hat{u}\left(j, k^{\prime}\right)-\hat{u}\left(j-1, k^{\prime}\right)+\hat{f}\left(j, k^{\prime}\right) \Leftrightarrow \\
& \Leftrightarrow v_{j+2}=a_{j} v_{j+1}+v_{j}-f_{j} .
\end{aligned}
$$


- Se $a=1, v_{j}=i^{j} \hat{u}\left(j-1, k^{\prime}\right) ; a_{j}=\frac{p\left(j^{2}\right)}{i} ; f_{j}=\hat{f}\left(j, k^{\prime}\right) . i^{j}$, donde:

$$
v_{j+2}=i^{j+2} \hat{u}\left(j+1, k^{\prime}\right) ; v_{j+1}=i^{j+1} \hat{u}\left(j, k^{\prime}\right)
$$

e (3.2) fica:

$$
\begin{gathered}
\hat{u}\left(j+1, k^{\prime}\right)=-p\left(j^{2}\right) \hat{u}\left(j, k^{\prime}\right)-\hat{u}\left(j-1, k^{\prime}\right)+\hat{f}\left(j, k^{\prime}\right) \Leftrightarrow \\
\Leftrightarrow \frac{v_{j+2}}{i^{j+2}}=-i a_{j} \frac{v_{j+1}}{i^{j+1}}-\frac{v_{j}}{i^{j}}+\frac{f_{j}}{i^{j}} \Leftrightarrow \\
\Leftrightarrow v_{j+2}=a_{j} v_{j+1}+v_{j}-f_{j}
\end{gathered}
$$

Inicialmente, resolveremos a equação de diferenças (3.3) para $j \geq 1$.

Definimos $p_{j}$ e $q_{j}$ como no lema (3.1) e vale:

$$
p_{j+1} q_{j}-p_{j} q_{j+1}=(-1)^{j}, j=1,2, \ldots
$$

Daí segue que:

$$
\frac{p_{j+1}}{q_{j+1}}-\frac{p_{j}}{q_{j}}=\frac{(-1)^{j}}{q_{j} q_{j+1}}
$$

e, usando esta fórmula recursivamente, chegamos à expressão:

$$
\frac{p_{j+1}}{q_{j+1}}=\frac{p_{2}}{q_{2}}+\sum_{r=2}^{j} \frac{(-1)^{r}}{q_{r} q_{r+1}}=\sum_{r=2}^{j} \frac{(-1)^{r}}{q_{r} q_{r+1}}, \text { pois } p_{2}=\mathbf{0} .
$$

Assim, definimos :

$$
t \equiv \lim _{j \rightarrow \infty} \frac{p_{j}}{q_{j}}=\sum_{r=2}^{\infty} \frac{(-1)^{r}}{q_{r} q_{r+1}}
$$

e, pelo lema (3.3), segue que $t$ está bem definido e existem constantes $K_{0}>0, j_{0}>0$, independentes de $j$, tais que:

$$
\left|p_{j}-t q_{j}\right| \leq K_{0}\left|q_{j}\right|^{-1}, j \geq j_{0} .
$$

Pela observação (3.2), temos que $t \neq 0$.

Atribuindo-se os valores iniciais: $v_{1}=\alpha ; v_{2}=\beta$ à equação (3.3) (os quais serão determinados posteriormente), sua solução geral será:

$$
v_{j}=\alpha p_{j}+\beta q_{j}+\varphi_{j}, j=1,2, \ldots,
$$


com:

$$
\varphi_{1}=\varphi_{2}=0 \text { e se } j \geq 3, \varphi_{j} \text { é dado como no lema (3.4) }
$$

Assim, podemos escrever:

$$
v_{j}=\alpha p_{j}+\left(\beta-\gamma_{1}\right) q_{j}+r_{j}
$$

onde $r_{j}=T_{1}+T_{2}-f_{j-2}=r_{j}\left(k^{\prime}\right)$ para cada $k^{\prime}$ fixado, e $T_{1}, T_{2}, \gamma_{1}$ são dados no lema (3.4).

Como $f \in C^{\infty}\left(T^{n}\right)$, temos que $f_{j-2}=\hat{f}\left(j-2, k^{\prime}\right)$ decresce rapidamente quando $j,\left|k^{\prime}\right| \rightarrow \infty$. Mostremos que $r_{j}\left(k^{\prime}\right)$ também tem decrescimento rápido quando $j,\left|k^{\prime}\right| \rightarrow \infty$, observando que $T_{1}, T_{2}$ assim se comportam.

Com efeito, do lema (3.3), temos:

$$
\left|p_{j}-t q_{j}\right| \leq K_{0}\left|q_{j}\right|^{-1}, j \geq j_{0}, K_{0}>0
$$

e tomando-se $\mu=[j / 2]+1$, vem:

$$
\begin{aligned}
\bullet\left|T_{1}\right|=\left|T_{1}\left(k^{\prime}\right)\right| & =\left|\left(p_{j}-t q_{j}\right) \cdot \sum_{\nu=1}^{j-3} f_{\nu}(-1)^{\nu} q_{\nu+1}\right| \\
& \leq\left|p_{j}-t q_{j}\right| \sum_{\nu=1}^{j_{3}}\left|f_{\nu}\right|\left|q_{\nu+1}\right| \\
& \stackrel{j \geq j_{0}}{\leq} K_{0}\left|q_{j}\right|^{-1}\left\{\sum_{\nu=1}^{\mu-1}+\sum_{\nu=\mu}^{j-3}\right\}\left(\left|f_{\nu}\right|\left|q_{\nu+1}\right|\right) \\
& \leq K_{0}\left|q_{j}\right|^{-1} \cdot\left|q_{\mu}\right| \sum_{\nu=1}^{\mu-1}\left|f_{\nu}\right|+K_{0} \max _{\nu \geq \mu}\left\{\left|f_{\nu}\right| \cdot \sum_{\nu=\mu}^{j-3} \frac{\left|q_{\nu+1}\right|}{\left|q_{j}\right|}\right\} .
\end{aligned}
$$

Pela observação (3.1), temos que $\exists K>1$ tal que:

$$
\left|q_{j}\right|=\left|q_{(j-\mu)+\mu}\right| \geq K^{(j-\mu)}\left|q_{\mu}\right| \Rightarrow\left|q_{j}\right|^{-1} \leq \frac{1}{K^{(j-\mu)} \mid q_{\mu}} \leq \frac{1}{k^{j / 2-1}\left|q_{\mu}\right|}
$$

Assim:

$$
K_{0}\left|q_{j}\right|^{-1}\left|q_{\mu}\right| \leq \frac{K_{0}}{K^{j / 2-1}}
$$

e ainda:

$$
\sum_{\nu=\mu}^{j-3} \frac{\left|q_{\nu+1}\right|}{\left|q_{j}\right|} \leq \sum_{\nu=\mu}^{j-3} \frac{\left|q_{j-2}\right|}{\left|q_{j}\right|} \leq \sum_{\nu=\mu}^{j-3} \frac{\left|q_{j-2}\right|}{K^{2}\left|q_{j-2}\right|} \leq \frac{j}{2 K^{2}}
$$


uma vez que esta soma envolve menos que $j / 2$ termos.

Logo:

$$
\left|T_{1}\right| \leq \frac{K_{0}}{K^{j / 2-1}} \cdot \sum_{\nu=1}^{\infty}\left|f_{\nu}\right|+\frac{K_{0}}{2 K^{2}} \max _{\nu \geq \mu}\left\{j \cdot\left|f_{\nu}\right|\right\}, \text { onde } f_{\nu}=\hat{f}\left(\nu, k^{\prime}\right) .
$$

onde $f_{\nu}=\hat{f}\left(\nu, k^{\prime}\right)$.

Então, $\forall r>0, \forall s>0$, teremos:

$$
\sup \left\{j^{s}\left|k^{\prime}\right|^{r}\left|T_{1}\right| ; j \in \mathbf{N} ; k^{\prime} \in \mathbf{Z}^{n-1}\right\} \leq
$$

$\leq \sup _{j \leq j_{0}}\left\{j^{s}\left|k^{\prime}\right|^{r}\left|T_{1}\right| ; k^{\prime} \in \mathbf{Z}^{n-1}\right\}+\sup _{j>j_{0}}\left\{\frac{K_{0}}{K^{j / 2-1}} j^{s} \cdot \sum_{\nu=1}^{\infty}\left|k^{\prime}\right|^{r}\left|\hat{f}\left(j, k^{\prime}\right)\right| ; k^{\prime} \in \mathbf{Z}^{n-1}\right\}+$

$$
+\frac{K_{0}}{2 K^{2}} \cdot \sup _{j>j_{0}}\left\{\left|k^{\prime}\right|^{r} \cdot \sup _{\nu \geq \mu} j^{s+1}\left|\hat{f}\left(\nu, k^{\prime}\right)\right| ; k^{\prime} \in \mathbf{Z}^{n-1}\right\}
$$

Mas, como $\mu=[j / 2]+1$, temos:

$$
\begin{gathered}
\left|\frac{j}{\mu}\right| \leq\left|\frac{j}{j / 2}\right|=2 \text { e } \\
j^{s+1}\left|\hat{f}\left(\mu, k^{\prime}\right)\right|=\left|\frac{j}{\mu}\right|^{s+1}|\mu|^{s+1}\left|\hat{f}\left(\mu, k^{\prime}\right)\right| \leq 2^{s+1}|\mu|^{s+1}\left|\hat{f}\left(\mu, k^{\prime}\right)\right| \\
j^{s+1}\left|\hat{f}\left(\mu+1, k^{\prime}\right)\right|=\left|\frac{j}{\mu+1}\right|^{s+1}|\mu+1|^{s+1}\left|\hat{f}\left(\mu+1, k^{\prime}\right)\right| \leq 2^{s+1}|\mu+1|^{s+1}\left|\hat{f}\left(\mu+1, k^{\prime}\right)\right| \\
\vdots \quad j|\hat{j}|^{s+1}|j-1|^{s+1}\left|\hat{f}\left(j-1, k^{\prime}\right)\right| \leq 2^{s+1}|j-1|^{s+1}\left|\hat{f}\left(j-1, k^{\prime}\right)\right| \\
j^{s+1}\left|\hat{f}\left(j-1, k^{\prime}\right)\right|=\left|\frac{j-1}{j+1}\right| \hat{f}\left(j, k^{\prime}\right)\left|\leq 2^{s+1} j^{s+1}\right| \hat{f}\left(j, k^{\prime}\right) \mid \\
(j+1)^{s+1}\left|\hat{f}\left(j+1, k^{\prime}\right)\right| \leq 2^{s+1}(j+1)^{s+1}\left|\hat{f}\left(j+1, k^{\prime}\right)\right|
\end{gathered}
$$


Donde concluímos que:

$$
j^{s+1}\left|\hat{f}\left(\nu, k^{\prime}\right)\right| \leq 2^{s+1}|\nu|^{s+1}\left|\hat{f}\left(\nu, k^{\prime}\right)\right|, \forall \nu \geq \mu .
$$

Daí:

$$
\begin{aligned}
\left.\left.\sup _{j, k^{\prime}}\left\{\left|k^{\prime}\right|^{r} \cdot \sup _{\nu \geq \mu}\left[j^{s+1} \mid \hat{f}\right) \nu, k^{\prime}\right) \mid\right]\right\} & \leq 2^{s+1} \sup _{j, k^{\prime}}\left\{\left|k^{\prime}\right|^{r} \cdot \sup _{\nu \geq \mu}\left[|\nu|^{s+1}\left|\hat{f}\left(\nu, k^{\prime}\right)\right|\right]\right\} \\
& =2^{s+1} \sup _{j, k^{\prime}}\left\{\sup _{\nu \geq[j / 2]+1}\left[\left|k^{\prime}\right|^{r}|\nu|^{s+1}\left|\hat{f}\left(\nu, k^{\prime}\right)\right|\right]\right\} \\
& =2^{s+1} \sup _{j, k^{\prime}}\left\{\left|k^{\prime}\right|^{r} j^{s+1}\left|\hat{f}\left(j, k^{\prime}\right)\right|\right\}<\infty
\end{aligned}
$$

uma vez que $f$ decresce rapidamente. Com isto, garantimos a limitação da terceira parcela em (3.6). A segunda parcela também é finita, pois $j /\left(K^{j / 2-1}\right)<M<\infty$ para todo $j$ e $\sum_{\nu=1}^{\infty}\left|k^{\prime}\right|^{r}\left|\hat{f}\left(\nu, k^{\prime}\right)\right|<\infty, \forall r>0$, pois $f \in C^{\infty}\left(T^{n}\right)$. Além disso, na primeira parcela temos:

$$
\begin{aligned}
& \sup _{j \leq j_{0}}\left\{j^{s}\left|k^{\prime}\right|{ }^{r}\left|T_{1}\right| ; k^{\prime} \in \mathbf{Z}^{n-1}\right\} \leq \\
& \leq \max _{j \leq j_{0}}\left\{j^{s}\left|q_{j_{0}-2}\right|\left|p_{j}-t q_{j}\right| \cdot \sup _{k^{\prime} \in \mathbf{Z}^{n-1}}\left\{\sum_{\nu=1}^{j-3}\left|k^{\prime}\right|{ }^{r}\left|\hat{f}\left(\nu, k^{\prime}\right)\right|\right\}\right\},
\end{aligned}
$$

pois $p_{j}$ e $q_{j}$ independem de $k^{\prime}$, para todo $j$ (os $a_{j}^{\prime} s$ são polinômios em $j$ ). E vemos que esta também é finita, uma vez que $f \in C^{\infty}\left(T^{n}\right) \operatorname{esup}_{k^{\prime} \in Z^{n}}\left\{\left|k^{\prime}\right|{ }^{r}\left|\hat{f}\left(\nu, k^{\prime}\right)\right|\right\}<\infty$. Com isto, concluímos que $T_{1}$ decresce rapidamente comforme $j,\left|k^{\prime}\right| \rightarrow \infty$.

O mesmo vale para $T_{2}$ :

$$
\begin{aligned}
& \bullet\left|T_{2}\right|=\left|T_{2}\left(k^{\prime}\right)\right|=\left|q_{j}\right|\left|\gamma_{1}-\sum_{\nu=1}^{j-3} f_{\nu \cdot}(-1)^{\nu}\left(p_{\nu+1}-t q_{\nu+1}\right)\right| \\
& =\left|q_{j}\right|\left|\sum_{\nu=j-2}^{\infty} f_{\nu} \cdot(-1)^{\nu}\left(p_{\nu+1}-t q_{\nu+1}\right)\right| \\
& \leq\left|q_{j}\right| \sum_{\nu=j-2}^{\infty}\left|f_{\nu}\right|\left|p_{\nu+1}-t q_{\nu+1}\right| \stackrel{j-2 \geq j_{0}}{\leq} K_{0} \sum_{\nu=j-2}^{\infty} \frac{\left|f_{\nu} \| q_{j}\right|}{\left|q_{\nu+1}\right|} \\
& =K_{0}\left(\frac{\left|q_{j}\right|}{\left|q_{j-1}\right|}\left|f_{j-2}\right|+\frac{\left|q_{j}\right|}{\left|q_{j}\right|}\left|f_{j-1}\right|+\frac{\left|q_{j}\right|}{\left|q_{j+1}\right|}\left|f_{j}\right|+\ldots\right)
\end{aligned}
$$


Mas:

$$
\begin{aligned}
\frac{\left|q_{j}\right|}{\left|q_{j-1}\right|} & \stackrel{s=j-1}{=} \frac{\left|q_{s+1}\right|}{\left|q_{s}\right|}=\frac{\left|a_{s-1} q_{s}+q_{s-1}\right|}{\left|q_{s}\right|} \\
& \leq\left|a_{s-1}\right|+\frac{\left|q_{s-1}\right|}{\left|q_{s}\right|} \leq\left|a_{s-1}\right|+1 \\
& \leq\left|p\left((s-1)^{2}\right)\right|+1 \leq C_{1}(s-1)^{2 m}=C_{1}(j-2)^{2 m}
\end{aligned}
$$

com $j \geq j_{1}$, para algum $j_{1}>0$ e alguma constante $C_{1}>0$.

Logo:

$$
\begin{aligned}
\left|T_{2}\left(k^{\prime}\right)\right| & \leq K_{0}\left(C_{1}(j-2)^{2 m}\left|f_{j-2}\right|+\left|f_{j-1}\right|+\left|f_{j}\right|+\cdots\right) \\
& \leq K_{0}\left(\tilde{C}_{1} j^{2 m}\left|f_{j-2}\right|+\left|f_{j-1}\right|+\cdots\right)
\end{aligned}
$$

donde, $\forall r>0, \forall s>0$, temos:

$$
\begin{gathered}
\sup \left\{j^{s}\left|k^{\prime}\right|^{r}\left|T_{2}\right| ; k^{\prime} \in \mathbf{Z}^{n-1} ; j \in \mathbf{N}\right\} \leq \\
\leq K_{0} \max _{j \leq j_{0}} \sup _{k^{\prime}}\left\{j^{s}\left|k^{\prime}\right|^{r}\left|T_{2}\right| ; k^{\prime} \in \mathbf{Z}^{n-1}\right\}+ \\
+K_{0} \sup _{j \geq j_{0}}\left\{\sup _{k^{\prime}}\left[\tilde{C}_{1} j^{s+2 m}\left|k^{\prime}\right|^{r}\left|\hat{f}\left(j-2, k^{\prime}\right)\right|\right\}+\right. \\
+K_{0} \sup _{j \leq j_{0}, k^{\prime}}\left\{j^{s}\left|k^{\prime}\right|^{r}\left|\hat{f}\left(j-1, k^{\prime}\right)\right|\right\}+K_{0} \sup _{j \geq j_{0}, k^{\prime}}\left\{\sum_{l=j}^{\infty} j^{s}\left|k^{\prime}\right|^{r}\left|\hat{f}\left(l, k^{\prime}\right)\right|\right\} \leq \\
\leq K_{0} \max _{j \leq j_{0}}\left\{j^{s}\left|q_{j}\right| \cdot \sup _{k^{\prime} \in \mathbf{Z}^{n-1}}\left[\sum_{\nu=j-1}^{\infty}\left|k^{\prime}\right|^{r}\left|\hat{f}\left(\nu, k^{\prime}\right)\right|\left|p_{\nu+1}-t q_{\nu+1}\right|\right]\right\}+ \\
+K_{0} \sup _{j \geq j_{0}, k^{\prime}}\left\{C_{2}(j-2)^{2 m+s}\left|k^{\prime}\right|^{r}\left|\hat{f}\left(j-2, k^{\prime}\right)\right|\right\}+ \\
+K_{0} \sup _{j \geq j_{0}, k^{\prime}}\left\{C_{3}(j-1)^{s}\left|k^{\prime}\right|^{r}\left|\hat{f}\left(j-1, k^{\prime}\right)\right|\right\}+K_{0} \sup _{k^{\prime}}\left\{\sum_{j=1}^{\infty} j^{s}\left|k^{\prime}\right|^{r}\left|\hat{f}\left(j, k^{\prime}\right)\right|\right\} .
\end{gathered}
$$

Observemos que a primeira parcela desta última desigualdade é finita, uma vez que temos um número finito de $j^{\prime} s$, os termos $q_{j}$ e $\left|p_{\nu+1}-t q_{\nu+1}\right|$ independem 
de $k^{\prime}$ para todo $\nu$, e este último é muito pequeno quando $j \geq j_{0}$, donde concluímos que :

$$
\sup _{k^{\prime}}\left\{\sum_{\nu=j-2}^{\infty}\left|k^{\prime}\right|^{r}\left|\hat{f}\left(\nu, k^{\prime}\right)\right|\left|p_{\nu+1}-t q_{\nu+1}\right|\right\}<\infty .
$$

A segunda e terceira parcelas também são limitadas, pois $f \in C^{\infty}\left(T^{n}\right)$; na quarta parcela podemos escrever:

$$
K_{0} \sup _{k^{\prime}}\left\{\sum_{j+1}^{\infty} j^{s}\left|k^{\prime}\right|^{r}\left|\hat{f}\left(j, k^{\prime}\right)\right|\right\} \leq K_{0} \sum_{j, k^{\prime}} j^{s}\left|k^{\prime}\right|^{\top}\left|\hat{f}\left(j, k^{\prime}\right)\right|=M<\infty
$$

devido ao decaimento rápido de $\hat{f}\left(j, k^{\prime}\right)$ quando $j,\left|k^{\prime}\right| \rightarrow \infty$. Com isto, verificamos o decrescimento rápido de $T_{2}$.

Agora, como $u \in \mathcal{D}^{\prime}\left(T^{n}\right), v_{j}$ deve ter, no máximo, crescimento polinomial quando $j \rightarrow \infty$. Logo, existem $s>0$ e $K>1$ (lema (3.2)) tais que:

$$
\frac{v_{j}}{q_{j}}=\alpha \frac{p_{j}}{q_{j}}+\left(\beta-\gamma_{1}\right) \frac{q_{j}}{q_{j}}+\frac{r_{j}}{q_{j}} \leq \frac{j^{s}}{K^{j-3}}, \text { se } j \geq 4
$$

Assim, temos:

$$
0 \leq \lim _{j \rightarrow \infty}\left|\alpha \frac{p_{j}}{q_{j}}+\left(\beta-\gamma_{1}\right)+\frac{r_{j}}{q_{j}}\right|=\left|\alpha t+\left(\beta-\gamma_{1}\right)\right| \leq \lim _{j \rightarrow \infty} \frac{j^{s}}{K^{j-3}}=0
$$

Portanto,

$$
\alpha t+\beta-\gamma_{1}=0
$$

A seguir, resolveremos a equação (3.3) para $j \leq 0$.

Como $v_{j+2}=a_{j} v_{j+1}+v_{j}-f_{j}, j=0, \pm 1, \pm 2, \ldots$ e valem as condições $(A .1)$, segue que:

$$
\begin{gathered}
v_{0}=v_{2}-a_{0} v_{1}+f_{0}, \quad \text { onde } a_{0}=p(0)=0 \mathrm{e} \\
v_{2}=\beta \Rightarrow v_{0}=\beta+f_{0}
\end{gathered}
$$

na equação (3.3), trocando $j$ por $(-j)$, temos:

$$
v_{-j}=-a_{-j} v_{1-j}+v_{2-j}+f_{-j}, j=1,2, \ldots
$$

e como $a_{-j}=a_{j}$, definimos:

$$
w_{j}=(-1)^{2-j} v_{2-j}
$$


e teremos:

$$
\begin{gathered}
v_{-j}=-a_{j} v_{1-j}+v_{2-j}+f_{-j}, j=1,2, \ldots \Leftrightarrow \\
\Leftrightarrow(-1)^{-j} w_{j+2}=-a_{j} .(-1)^{1-j} w_{j+1}+(-1)^{2-j} w_{j}+f_{-j} \Leftrightarrow \\
w_{j+2}=a_{j} w_{j+1}+w_{j}+(-1)^{j} f_{-j}, \quad j=1,2, \ldots
\end{gathered}
$$

e, assim, obtemos:

$$
w_{j+2}=a_{j} w_{j+1}+w_{j}-g_{j}, \operatorname{com} g_{j}=(-1)^{j+1} f_{-j}, j=1,2, \ldots,
$$

com valores iniciais: $w_{1}=\alpha^{\prime}=-v_{1}=-\alpha ; w_{2}=\beta^{\prime}=v_{0}=\beta+f_{0}$.

Pelo lema (3.4), uma solução para esta equação é:

$$
w_{j}=\alpha^{\prime} p_{j}+\beta^{\prime} q_{j}+\varphi_{j}=-\alpha p_{j}+\left(\beta+f_{0}\right) q_{j}+\varphi_{j}, j=1,2, \ldots
$$

onde

$$
\begin{aligned}
& \varphi_{1}=\varphi_{2}=0 \text { e para } j \geq 3: \\
& \varphi_{j}=\left(p_{j}-t q_{j}\right) \sum_{\nu=1}^{j-3} g_{\nu} \cdot(-1)^{\nu} q_{\nu+1}+q_{j}\left\{\gamma_{2}-\sum_{\nu=1}^{j-3} g_{\nu} \cdot(-1)^{\nu}\left(p_{\nu+1}-t q_{\nu+1}\right)\right\}- \\
& -g_{j-2}-\gamma_{2} q_{j}= \\
& =\left(p_{j}-t q_{j}\right) \sum_{\nu=1}^{j-3}(-1)^{\nu} f_{-\nu} \cdot(-1)^{\nu} q_{\nu+1}+q_{j}\left\{\gamma_{2}-\sum_{\nu=1}^{j-3}(-1)^{2 \nu} f_{-\nu}\left(p_{\nu+1}-t q_{\nu+1}\right\}-\right. \\
& -(-1)^{j-1} f_{-(j-2)}-\gamma_{2} q_{j}= \\
& =\left(p_{j}-t q_{j}\right) \sum_{\nu=1}^{j-3} f_{-\nu} q_{\nu+1}+q_{j}\left\{\gamma_{2}-\sum_{\nu=1}^{j-3} f_{-\nu}\left(p_{\nu+1}-t q_{\nu+1}\right)\right\}+ \\
& +(-1)^{j-1} f_{2-j}-\gamma_{2} q_{j} \equiv \tilde{T}_{1}+\tilde{T}_{2}+(-1)^{j} f_{2-j}-\gamma_{2} q_{j},
\end{aligned}
$$

onde

$$
\gamma_{2}=\sum_{\nu=1}^{\infty} f_{-\nu}\left(p_{\nu+1}-t q_{\nu+1}\right)
$$

Observamos que $\gamma_{2}$ e

$$
\tilde{T}_{1}=\left(p_{j}-t q_{j}\right) \sum_{\nu=1}^{j-3} f_{-\nu} q_{\nu+1} ; \tilde{T}_{2}=q_{j}\left\{\gamma_{2}-\sum_{\nu=1}^{j-3} f_{-\nu}\left(p_{\nu+1}-t q_{\nu+1}\right)\right\}
$$

são expressões idênticas a $\gamma_{1}, T_{1}$ e $T_{2}$, a menos do fator $(-1)^{\nu}$ em cada parcela do somatório. Assim, podemos concluir que $\tilde{T}_{1}$ e $\tilde{T}_{2}$ também decrescem rapidamente quando $j,\left|k^{\prime}\right| \rightarrow \infty$. 
Logo, $w_{j}=-\alpha p_{j}+\left(\beta+f_{0}+\gamma_{2}\right) q_{j}+\tilde{r_{j}}$, onde $\tilde{r_{j}}=\tilde{T}_{1}+\tilde{T}_{2}+(-1)^{j} f_{2-j}$, decresce rapidamente quando $j,\left|k^{\prime}\right| \rightarrow \infty$.

Como $u \in \mathcal{D}^{\prime}\left(T^{n}\right), w_{j}$ tem, no máximo, crescimento polinomial quando $j \rightarrow \infty$, donde segue que existem $s>0$ e $K>1$ (lema 3.2) tais que:

$$
\left|\frac{w_{j}}{q_{j}}\right|=\left|-\alpha \frac{p_{j}}{q_{j}}+\left(\beta+f_{0}+\gamma_{2}\right)+\frac{\tilde{r}_{j}}{q_{j}}\right| \leq \frac{j^{s}}{\left|q_{j}\right|} \leq \frac{j^{s}}{K^{j-2}}
$$

Logo,

$$
\lim _{j \rightarrow \infty}\left[-\alpha \frac{p_{j}}{q_{j}}+\left(\beta+f_{0}+\gamma_{2}\right)+\frac{\tilde{r}_{j}}{q_{j}}\right]=-\alpha t+\beta+f_{0}+\gamma_{2}=0
$$

Combinando (3.7) e (3.8), segue que:

$$
\alpha=\left(\gamma_{1}+\gamma_{2}+f_{0}\right) /(2 t) ; \quad \beta=\left(\gamma_{1}-\gamma_{2}-f_{0}\right) / 2 .
$$

Podemos ver facilmente que $\alpha$ e $\beta$ independem de $j$, pois $\gamma_{1}=\gamma_{1}\left(k^{\prime}\right)$; $\gamma_{2}=\gamma_{2}\left(k^{\prime}\right)$ e $f_{0}=\hat{f}\left(0, k^{\prime}\right)$ ( $t$ é um número fixo e bem determinado); além disso, estes têm decrescimento rápido quando $\left|k^{\prime}\right| \rightarrow \infty$. Para isto, basta observarmos que $\gamma_{1}, \gamma_{2}$ e $f_{0}$ assim se comportam.

Com efeito:

$$
\begin{aligned}
\left|\gamma_{1}\left(k^{\prime}\right)\right| & \leq \sum_{\nu=1}^{\infty}\left|\hat{f}\left(\nu, k^{\prime}\right)\right|\left|p_{\nu+1}-t q_{\nu+1}\right| \leq \sup _{\nu \in \mathbb{N}}\left|\hat{f}\left(\nu, k^{\prime}\right)\right| \cdot \sum_{\nu=1}^{\infty}\left|p_{\nu+1}-t q_{\nu+1}\right| \\
& \leq \sup _{\nu \in \mathbb{N}}\left|\hat{f}\left(\nu, k^{\prime}\right)\right| \cdot\left\{\sum_{\nu=1}^{j_{0}-1}\left|p_{\nu+1}-t q_{\nu+1}\right|+\sum_{\nu=j_{0}}^{\infty} \frac{K_{0}}{K^{\nu+1-3}}\right\} \\
& \leq \sup _{\nu \in \mathbb{N}}\left|\hat{f}\left(\nu, k^{\prime}\right)\right|,\left\{C_{1}+\sum_{\nu=j_{0}}^{\infty} \frac{K_{0}}{K^{\nu-2}}\right\},
\end{aligned}
$$

onde a última soma converge, pois $K>1$, e podemos escrever:

$$
\begin{gathered}
\left|\gamma_{1}\left(k^{\prime}\right)\right| \leq M \sup _{\nu}\left|\hat{f}\left(\nu, k^{\prime}\right)\right|, \text { com } \\
M=C_{1}+\sum_{\nu=j_{0}}^{\infty} \frac{K_{0}}{K^{\nu-2}}<\infty .
\end{gathered}
$$

Assim, $\forall r>0$, temos:

$$
\sup _{k^{\prime} \in \mathbf{Z}^{n-1}}\left\{\left|k^{\prime}\right|^{r}\left|\gamma_{1}\left(k^{\prime}\right)\right|\right\} \leq M \sup _{\nu, k^{\prime}}\left\{\left|k^{\prime}\right|^{r}\left|\hat{f}\left(\nu, k^{\prime}\right)\right|\right\}<\infty,
$$


pois $f$ decresce rapidamente.

$\mathrm{O}$ decrescimento rápido de $\gamma_{2}$ é mostrado da mesma maneira.

Afirmamos que, sob tais condições, $v_{j}, j \geq 1$ decresce rapidamente quando $j,\left|k^{\prime}\right| \rightarrow \infty$.

De fato:

$$
v_{j}=\alpha p_{j}+\left(\beta-\gamma_{1}\right) q_{j}+T_{1}+T_{2}-f_{j-2}
$$

e já sabemos que $T_{1}, T_{2}, f_{j-2}$ decrescem rapidamente quando $j,\left|k^{\prime}\right| \rightarrow \infty$.

Assim, resta verificarmos o termo $\left(\alpha p_{j}+\left(\beta-\gamma_{1}\right) q_{j}\right)$. Para tanto, temos:

$$
\left|\alpha \frac{p_{j}}{q_{j}}+\left(\beta-\gamma_{1}\right)\right|=\left|\alpha \frac{p_{j}}{q_{j}}-\alpha t+\left(\alpha t+\beta-\gamma_{1}\right)\right| \stackrel{(3.7)}{=}|\alpha|\left|\frac{p_{j}}{q_{j}}-t\right|
$$

Daí:

$$
\left|\alpha p_{j}+\left(\beta-\gamma_{1}\right) q_{j}\right|=|\alpha|\left|p_{j}-t q_{j}\right| \stackrel{j \geq j_{1} \geq 4}{\leq}|\alpha| \frac{K_{0}}{\left|q_{j}\right|} \leq|\alpha| \frac{K_{0}}{K^{j-2}} .
$$

Logo, dados $r>0, s>0$, teremos:

$$
\sup _{j, k^{\prime}}\left\{j^{s}\left|k^{\prime}\right|^{r}\left|\alpha p_{j}+\left(\beta-\gamma_{1}\right) q_{j}\right|\right\} \leq K_{0} \sup _{j, k^{\prime}}\left\{|\alpha|\left|k^{\prime}\right|^{r} \frac{j^{s}}{K^{j-2}}\right\}<\infty,
$$

uma vez que $\alpha$ decai rapidamente quando $\left|k^{\prime}\right| \rightarrow \infty$ e $\sup _{j \in \mathbb{N}} \frac{j^{s}}{K^{j-2}}<\infty$.

Do mesmo modo, podemos ver que $w_{j}, j \geq 0$ decresce rapidamente quando $j,\left|k^{\prime}\right| \rightarrow \infty$ :

$$
\begin{aligned}
\frac{w_{j}}{q_{j}} & =-\alpha \frac{p_{j}}{q_{j}}+\left(\beta+f_{0}+\gamma_{2}\right)+\frac{\tilde{r_{j}}}{q_{j}} \\
& =-\alpha\left(\frac{p_{j}}{q_{J}}-t\right)-\alpha t+\beta+f_{0}+\gamma_{2}+\frac{\tilde{r_{j}}}{q_{j}}
\end{aligned}
$$

e, como $-\alpha t+\beta+f_{0}+\gamma_{2}=0$ (eq.(3.8)), temos:

$$
w_{j}=-\alpha\left(p_{j}-t q_{j}\right)+\tilde{r_{j}} \Rightarrow\left|w_{j}\right| \leq|\alpha| \frac{K_{0}}{\left|q_{j}\right|}+\left|\tilde{r}_{j}\right|
$$

e já vimos que $\tilde{r_{j}}$ decresce rapidamente quando $j,\left|k^{\prime}\right| \rightarrow \infty$ e o termo $|\alpha| \frac{K_{0}}{\left|q_{j}\right|}$ assim se comporta, como foi observado anteriormente. 
Com isso, temos: $w_{j}=(-1)^{2-j} v_{2-j}$ e vemos que $v_{-j}$ também tem decrescimento rápido quando $j,\left|k^{\prime}\right| \rightarrow \infty$. Portanto, $\hat{u}\left(j, k^{\prime}\right)=v_{j+1}, j=0, \pm 1, \pm 2, \ldots$ também decresce rapidamente, donde concluímos, finalmente, que $u \in C^{\infty}\left(T^{n}\right)$ e o operador $P$ é $(\mathrm{GH})$.

\subsection{Observações relevantes sobre o teorema central}

\subsubsection{A unicidade da solução}

Sob as hipóteses $(A .1)$ da seção (3.1), pode-se mostrar que a solução $u \in \mathcal{D}^{\prime}\left(T^{n}\right)$ da equação

$$
P u=\left(p\left(D_{1}^{2}\right)+e^{i x_{1}}+a e^{-i x_{1}}\right) u=f
$$

é única.

De fato: se $P u=f$, então, vimos que:

$$
a \hat{u}\left(k+e_{1}\right)=-p\left(k_{1}^{2}\right) \hat{u}(k)-\hat{u}\left(k-e_{1}\right)+\hat{f}(k),
$$

onde $e_{1}=(1,0, \ldots, 0) \in \mathbf{Z}^{n}$, e tal expressão pode ser reduzida à equação:

$$
v_{j+2}=a_{j} v_{j+1}+v_{j}-f_{j}, \quad j=0, \pm 1, \pm 2, \ldots
$$

Com as condições $v_{1}=\alpha ; v_{2}=\beta$, existe um único par $(\alpha, \beta)$ de forma que $u \in \mathcal{D}\left(T^{n}\right)$. Com isto, os $v_{j}^{\prime} s$ são determinados de maneira única por $\alpha, \beta$ e pelos coeficientes de Fourier de $f$. Assim, os $\hat{u}\left(j, k^{\prime}\right)^{\prime} s$ também são únicos para todo $\left(j, k^{\prime}\right)$ (pois $\hat{u}\left(j, k^{\prime}\right)=v_{j+1} /(\sqrt{-a})^{j}, a= \pm 1$ ) e daí segue a unicidade da solução $u$.

\subsubsection{O operador $P=D_{1}^{2}+2 r i \operatorname{sen} x_{1}, \operatorname{com} r>1$}

Este operador pode ser escrito como

$$
P=r\left(\frac{D_{1}^{2}}{r}+2 i \operatorname{sen} x_{1}\right)
$$


e será $(\mathrm{GH})$ se

$$
\tilde{P}=\frac{D_{1}^{2}}{r}+2 i \operatorname{sen} x_{1}
$$

o for.

Mas, como não podemos garantir que o polinômio $\tilde{p}(t)=\frac{t}{r}$ satisfaça a todas as hipóteses em ( $A .1)$, não podemos aplicar diretamente o teorema (3.1). Porém, tratando-se de um caso bastante particular, veremos que a demonstração vista em (3.3) continua válida, pois podemos obter aquelas desigualdades de outra maneira.

Com efeito, substituindo as expansões em séries de Fourier na equação $P u=f$, temos:

$$
r\left[\sum_{k} \frac{k_{1}^{2}}{r} \hat{u}(k) e^{i k . x}+\sum_{k} \hat{u}(k) e^{i\left(k_{1}+1, k^{\prime}\right) \cdot x}+\sum_{k} \hat{u}(k) e^{i\left(k_{1}-1, k^{\prime}\right) . x}\right]=\sum_{k} \hat{f}(k) e^{i k . x},
$$

donde:

$$
\frac{k_{1}^{2}}{r} \hat{u}(k)+\hat{u}\left(k-e_{1}\right)+\hat{u}\left(k+e_{1}\right)=\frac{\hat{f}(k)}{r},
$$

$\operatorname{com} e_{1}=(1,0, \ldots, 0) \in \mathbf{Z}^{n}, k^{\prime} \in \mathbf{Z}^{n-1}$.

Para cada $k^{\prime} \in \mathbf{Z}^{n-1}$, fazemos:

$$
k_{1}=j ; v_{j}=\hat{u}\left(j-1, k^{\prime}\right) ; a_{j}=\frac{j^{2}}{r} \text { e } f_{j}=\frac{\hat{f}\left(j, k^{\prime}\right)}{r}
$$

e a equação anterior fica:

$$
v_{j+2}=a_{j} v_{j+1}+v_{j}-f_{j}, j=0, \pm 1, \pm 2, \ldots
$$

Definimos $p_{j}$ e $q_{j}$ como no lema (3.1) e assim, continua válida a igualdade:

$$
p_{j+1} q_{j}-p_{j} q_{j+1}=(-1)^{j}, \quad j=1,2, \ldots
$$

Podemos ver que uma desigualdade análoga à do lema (3.2) também se verifica. De fato, basta observarmos que: 


\begin{tabular}{|l|l|}
\hline$q_{1}=0 ; q_{2}=1$ & $p_{1}=1 ; p_{2}=0$ \\
$q_{3}=1 / r>0$ & $p_{3}=1>0$ \\
$q_{4}=(4 / r) .(1 / r)+1>0$ & $p_{4}=4 / r>0$ \\
$q_{5}=(9 / r)\left(4 / r^{2}+1\right)>0$ & $p_{5}=(9 / r)(4 / r)+1>0$ \\
$\vdots$ & $\vdots$ \\
$q_{j} \geq 0, \forall j=1,2, \ldots$ & $p_{j} \geq 0, \forall j=1,2, \ldots$ \\
\hline
\end{tabular}

Tomemos $j_{0} \in \mathbf{N}$ tal que $q_{j_{0}+1}>1 ; p_{j_{0}+1}>1$ e $j_{0}>(r+1)$.

Para $j \geq j_{0}$, temos:

$$
a_{j}=\frac{j^{2}}{r} \geq j \frac{j_{0}}{r} \geq j \frac{r+1}{r}>j
$$

Assim,

$$
\begin{gathered}
q_{j_{0}+2}=a_{j_{0}} q_{j_{0}+1}+q_{j_{0}}>a_{j_{0}} q_{j_{0}+1}>j_{0} q_{j_{0}+1}>j_{0}>r \\
q_{j_{0}+3}=a_{j_{0}+1} q_{j_{0}+2}+q_{j_{0}+1}>a_{j_{0}+1} q_{j_{0}+2}>\left(j_{0}+1\right) j_{0}>j_{0}^{2}
\end{gathered}
$$

Portanto,

$$
q_{j_{0}+s}>r^{j_{0}+s-\left(j_{0}+1\right)}=r^{s-1}, s \geq 2
$$

e assim:

$$
\left|q_{j}\right|=q_{j}>r^{j-j_{0}-1}, \forall j \geq j_{0}+2
$$

Analogamente, podemos mostrar que:

$$
\left|p_{j}\right|=p_{j}>r^{j-j_{0}-1}, \forall j \geq j_{0}+2, \text { com } r>1 .
$$

Ainda, assim como na demonstração da seção (3.3), vale:

$$
\frac{p_{j+1}}{q_{j+1}}=\sum_{l=2}^{j} \frac{(-1)^{l}}{q_{l} q_{l+1}}
$$

e esta soma converge quando $j \rightarrow \infty$.

$$
\text { Definimos } t=\lim _{j \rightarrow \infty} \frac{p_{j}}{q_{j}}=\sum_{j=2}^{\infty} \frac{(-1)^{j}}{q_{j} q_{j+1}}
$$


e com as desigualdades verificadas acima, analogamente ao que foi feito no lema (3.3), podemos mostrar que existem $K_{0}>0$ e $j_{0} \geq 1$, independentes de $j$, tais que:

$$
\left|p_{j}-t q_{j}\right| \leq \frac{K_{0}}{\left|q_{j}\right|}, j \geq j_{0}
$$

e concluímos que $t \neq 0$, como antes.

Fazendo $v_{1}=\alpha ; v_{2}=\beta$, a solução para a equação:

$$
\begin{cases}v_{j+2}=a_{j} v_{j+1}+v_{j}-f_{j}, & j \geq 1 \\ v_{1}=\alpha ; & v_{2}=\beta\end{cases}
$$

é dada pelo lema (3.4).

Novamente podemos escrever:

$$
v_{j}=\alpha p_{j}+\left(\beta-\gamma_{1}\right) q_{j}+r_{j}
$$

e vemos que $r_{j}$ decresce rapidamente quando $j,\left|k^{\prime}\right| \rightarrow \infty$ e $\gamma_{1}$ assim se comporta quando $\left|k^{\prime}\right| \rightarrow \infty$, da mesma maneira que foi visto na seção (3.3).

Do fato de $v_{j}$ ter crescimento, no máximo polinomial, segue que $\alpha t+\beta-\gamma_{1}=0$ e, resolvendo a equação $v_{j+2}=a_{j} v_{j+1}+v_{j}-f_{j}$ para $j \leq 0$, novamente concluímos que $-\alpha t+\beta+f_{0}-\gamma_{2}=0$ e determinamos $\alpha$ e $\beta$.

Os mesmos argumentos da seção (3.3) são válidos para mostrar que $\alpha$ independe de $j$ e decai rapidamente quando $\left|k^{\prime}\right| \rightarrow \infty$. Portanto, da mesma forma, podemos provar que $\hat{u}\left(j, k^{\prime}\right)$ tem decrescimento rápido quando $j,\left|k^{\prime}\right| \rightarrow \infty$.

Observemos que esta prova foi possível devido às características peculiares do operador. O fato do polinômio envolvido ser da forma $p\left(D_{1}^{2}\right)=D_{1}^{2}$ e o aparecimento do termo 2 risen $x_{1}$ foram imprescindiveis para as estimativas:

$$
\left|p_{j}\right|>r^{j-j_{0}-1} \text { e }\left|q_{j}\right|>r^{j-j_{0}-1}, j \geq j_{0}+2 .
$$

Se tivéssemos o operador:

$$
P=D_{1}^{2}+2 r \cos x_{1}, r>1,
$$

surgiria a unidade imaginária na definição $a_{j}=j^{2} /(r \sqrt{-1})=j^{2} /(r i)$ e o argumento utilizado aqui para estimar os $p_{j}^{\prime} s$ e $q_{j}^{\prime} s$ já não seria válido, uma vez que 
as sequências obtidas seriam:

$$
\begin{array}{|l|l|}
\hline q_{1}=0 ; q_{2}=1 & p_{1}=1 ; p_{2}=0 \\
q_{3}=1 /(r i) & p_{3}=1 \\
q_{4}=1-4 /\left(r^{2}\right) & p_{4}=4 /(r i) \\
q_{5}=10 /(r i)-36 /\left(r^{3} i\right) & p_{5}=-36 /\left(r^{2}\right)+1 \\
\vdots & \vdots \\
\hline
\end{array}
$$

$\mathrm{E}$, neste caso, não teriamos a condição $p_{j}, q_{j} \geq 0$ que foi usada nas estimativas para o caso do operador anterior. Assim, este método, tal como está, ainda não é capaz de garantir que o operador $P=D_{1}^{2}+2 r \cos x_{1}, \operatorname{com} r>1$ seja (GH) em $T^{2}$.

\subsubsection{Por que utilizar grau par - ou $p\left(D_{1}^{2}\right)$}

Uma pergunta que surge imediatamente ao observarmos 0 operador $P=p\left(D_{1}^{2}\right)+e^{i x_{1}}+a e^{-i x_{1}}$, com $p(t)$ satisfazendo às condições:

$$
(A .2) \quad p(0)=0 ;|p(t)|>2,|t| \geq 2, t \in \mathbf{Z}
$$

é: " Por que não analisamos a hipoeliticidade global do operador:

$$
\tilde{P}=p\left(D_{1}\right)+e^{i x_{1}}+a e^{-i x_{1}},
$$

mantendo as propriedades ( $A .2)$ em $p$, da mesma maneira que na seção (3.3)?"

Se verificarmos a demostração (3.3), veremos que foi de extrema importância o fato de $a_{j}=a_{-j}=p\left(j^{2}\right) / \sqrt{-a}$ para a obtenção das duas equações:

$$
\left\{\begin{array}{l}
\alpha t+\beta-\gamma_{1}=0 \\
-\alpha t+\beta+f_{0}-\gamma_{2}=0
\end{array}\right.
$$

com as quais foi possível determinar um $\operatorname{par}(\alpha, \beta)$ único, em termos de $t, \gamma_{1}, \gamma_{2} e f_{0}$.

Analisando o caso de um polinômio $p$ ímpar, ou seja, $p(-t)=-p(t)$ e que ainda satisfaz $(A .2)$, vemos que a demonstração da seção (3.3) não é viável. 
De fato, temos: $P=p\left(D_{1}\right)+e^{i x_{1}}+a e^{-i x_{1}}$ e definimos:

$$
a_{j}=\frac{p(j)}{\sqrt{-a}} .
$$

Logo,

$$
a_{-j}=\frac{-p(j)}{\sqrt{-a}}=-a_{j}
$$

e, se vale ( $A .2)$, chegamos a uma equação da forma:

$$
\begin{gathered}
w_{j+2}=a_{j} w_{j+1}+w_{j}+f_{-j}, \quad j=1,2, \ldots \\
w_{1}=\alpha ; w_{2}=\beta+f_{0} .
\end{gathered}
$$

Pelo fato de $u \in \mathcal{D}^{\prime}\left(T^{n}\right)$, devemos ter: $\alpha t+\beta-\gamma_{1}=0$ e $\alpha t+\beta+\gamma_{2}+f_{0}=0$ e com estas equações não é possível determinar $\alpha$ e $\beta$.

Uma outra tentativa seria definir novos $p_{j}^{\prime}$ e $q_{j}^{\prime}$ para resolver a equação de diferenças quando $j \leq 0$. Com isto, teríamos um novo $t^{\prime}$ e duas equações:

$$
\alpha t+\beta-\gamma_{1}=0 e-\alpha t^{\prime}+\beta+f_{0}+\gamma_{2}=0 .
$$

Porém, uma análise rápida dos novos $p_{j}^{\prime} s$ e $q_{j}^{\prime} s$ mostra que

$$
\frac{p_{j}^{\prime}}{q_{j}^{\prime}}=-\frac{p_{j}}{q_{j}}
$$

e assim, $t^{\prime}=-t$. Logo, recaímos no mesmo problema.

Na verdade, podemos ver que o operador: $P=D_{1}-\gamma\left(x_{1}\right)$, onde $\gamma \in C_{0}^{\infty}\left(T^{1}\right)$ (i.e., $\int_{0}^{2 \pi} \gamma(t) d t=0$ ), não é (GH) em $T^{2}$, e com isso, $P=D_{1}+e^{i x_{1}}+a e^{-i x_{1}}$ também não é (GH).

De fato, existe $h \in C_{0}^{\infty}\left(T^{1}\right)$ tal que $D_{1} h=\gamma$, pois:

$$
D_{1} h=\frac{1}{i} \frac{\partial}{\partial t} h(t)=\gamma(t) \Rightarrow h(t)=i \int_{0}^{t} \gamma(s) d s, t \in[0,2 \pi] .
$$

É claro que $h \in C^{\infty}\left(\gamma \in C^{\infty}\right)$. Além disso,

$$
h(2 \pi)=i \int_{0}^{2 \pi} \gamma(s) d s=0=h(0)
$$


donde vemos que $h$ será $2 \pi$-periódica se definirmos

$$
h(t+2 k \pi)=h(t), k \in \mathbf{Z}, t \in[0,2 \pi] .
$$
temos:

Observemos, agora, que: para $u=u(t, x) \in \mathcal{D}^{\prime}\left(T^{2}\right)$ e $v(t, x)=e^{-h(t)} u(t, x)$,

$$
\begin{aligned}
D_{1} v(t, x) & =D_{1}\left(e^{-h(t)} u(t, x)\right) \\
& =D_{1}\left(e^{-h(t)}\right) u(t, x)+e^{-h(t)} D_{1} u(t, x) \\
& =-D_{1} h e^{-h} u+e^{-h} D_{1} u=e^{-h}\left[D_{1} u-\gamma u\right]=e^{-h} \cdot P u .
\end{aligned}
$$

Com isto, concluímos que analisar a hipoeliticidade global do operador $P$ é equivalente a analisar o operador $D_{1}$, uma vez que a equação $P u=f$ equivale a $D_{1} v=g$, com $g=e^{-h} f$. E, como $D_{1}$ não é $(\mathrm{GH})$ em $T^{2}$, vemos que $P$ também não.

\subsection{A hipoeliticidade global para o operador $Q=q\left(D^{\prime}\right)+2 \cos x_{1}$}

Apresentamos agora, um outro resultado sobre hipoeliticidade global, cuja prova será feita nos moldes da seção (3.3):

Teorema 3.2 Seja $q\left(\lambda^{\prime}\right)$ um polinômio de grau $m \geq 1$, onde $\lambda^{\prime}=\left(\lambda_{2}, \ldots, \lambda_{n}\right) \in \mathbf{R}^{n-1}$. Considere o operador:

$$
\begin{gathered}
Q=q\left(D^{\prime}\right)+2 \cos x_{1}, \text { onde } \\
q\left(D^{\prime}\right)=\sum_{\substack{a \in Z^{n-1} \\
|a| \leq m}} c_{\alpha} D_{2}^{\alpha_{2}} \ldots D_{n}^{\alpha_{n}}, D_{j}=\frac{1}{i} \frac{\partial}{\partial x_{j}}, j=2, \ldots, n .
\end{gathered}
$$

Suponha que exista $\tau>0$ tal que $\left|q\left(\lambda^{\prime}\right)\right| \geq 2+\tau$ para todo $\lambda^{\prime} \in \mathbf{Z}^{n-1}$. Então $Q$ é $(G H)$ em $T^{n}$.

Antes de demonstrarmos o teorema, analisemos alguns exemplos: 


\subsubsection{Exemplo 1}

Tome

$$
Q=-\frac{\partial^{2}}{\partial x_{2}^{2}}+3+2 \cos x_{1}
$$

e podemos escrever:

$$
Q=D_{2}^{2}+\gamma\left(x_{1}\right), \operatorname{com} \gamma\left(x_{1}\right)=3+2 \cos x_{1} \geq 1, \forall x_{1} \text {. }
$$

É fácil ver que $Q$ não é (H). Basta tomar:

$$
u\left(x_{1}, x_{2}\right)= \begin{cases}e^{x_{2} \sqrt{\gamma\left(x_{1}\right)},} & \text { se } x_{1}>0 \\ 0, & \text { se } x_{1} \leq 0\end{cases}
$$

e vemos que $u \in \mathcal{D}^{\prime}\left(\mathbf{R}^{2}\right) \backslash C^{\infty}\left(\mathbf{R}^{2}\right)$. (u pode ser escrito como: $u=e^{x_{2} \sqrt{\gamma\left(x_{1}\right)}} \cdot H\left(x_{1}\right)$, onde $H$ é a função de Heaviside:

$$
H\left(x_{1}\right)= \begin{cases}1, & \text { se } x_{1}>0 \\ 0, & \text { se } x_{1} \leq 0\end{cases}
$$

e $u$ não é contínua em $\left\{\left(0, x_{2}\right) ; x_{2} \in \mathbf{R}\right\}$. )

Assim, calculando as derivadas no sentido de distribuições, teremos:

$$
Q u=\left[D_{2}^{2}+\gamma\left(x_{1}\right)\right] u= \begin{cases}D_{2}\left(\frac{1}{i} \sqrt{\gamma\left(x_{1}\right)} e^{x_{2}} \sqrt{\gamma\left(x_{1}\right)}\right)+\gamma\left(x_{1}\right) e^{x_{2} \sqrt{\gamma\left(x_{1}\right)}} & \text { se } x_{1}>0 \\ 0, & \text { se } x_{1}<0\end{cases}
$$

Ainda, se $x_{1}>0$,

$$
\begin{aligned}
Q u & =D_{2}\left(\frac{1}{i} \sqrt{\gamma\left(x_{1}\right)} e^{x_{2} \sqrt{\gamma\left(x_{1}\right)}}\right)+\gamma\left(x_{1}\right) e^{x_{2} \sqrt{\gamma\left(x_{1}\right)}} \\
& =-\frac{\partial}{\partial x_{2}}\left[\sqrt{\gamma\left(x_{1}\right)} e^{x_{2} \sqrt{\gamma\left(x_{1}\right)}}\right]+\gamma\left(x_{1}\right) e^{x_{2} \sqrt{\gamma\left(x_{1}\right)}} \\
\stackrel{\gamma\left(x_{1}\right)>0}{=} & \left(-\gamma\left(x_{1}\right)+\gamma\left(x_{1}\right)\right) e^{x_{2} \sqrt{\gamma\left(x_{1}\right)}}=0
\end{aligned}
$$

e, portanto:

$$
Q u=0, \text { com } u \in \mathcal{D}^{\prime}\left(\mathbf{R}^{2}\right) \backslash C^{\infty}\left(\mathbf{R}^{2}\right)
$$

Mas o teorema (3.2) garante a hipoeliticidade global de $Q$ em $T^{2}$, uma vez que

$$
\left|q\left(x_{2}\right)\right|=\left|x_{2}^{2}+3\right| \geq 3=2+1(\tau=1) .
$$




\subsubsection{Exemplo 2}

Seja

$$
Q=-\left(\frac{\partial}{\partial x_{2}}\right)^{2}-\left(\frac{\partial}{\partial x_{3}}\right)^{2}+r(2+\tau) i+2 \cos x_{1}
$$

onde $\tau, r \in \mathbf{R}, r \geq 1, \tau>0$ e $i=\sqrt{-1}$.

Podemos escrever: $Q=D_{2}^{2}+D_{3}^{2}+r(2+\tau) i+2 \cos x_{1}$ e temos:

$$
\begin{gathered}
q\left(\lambda_{2}, \lambda_{3}\right)=\lambda_{2}^{2}+\lambda_{3}^{2}+r(2+\tau) i \Rightarrow \\
\left|q\left(\lambda_{2}, \lambda_{3}\right)\right| \geq r(2+\tau) \geq 2+\tau, \forall\left(\lambda_{2}, \lambda_{3}\right) \in \mathbf{Z}^{2} .
\end{gathered}
$$

Logo, $Q$ é (GH) em $T^{2}$.

Vemos, com este exemplo, que uma perturbação do operador

$$
\tilde{Q}=D_{2}^{2}+D_{3}^{2}+2 \cos x_{1}
$$

com o fator $r i(2+\tau), r \geq 1$, pode torná-lo $(\mathrm{GH})$, sem que seja preciso analisar a hipoeliticidade global de $\tilde{Q}$.

\subsubsection{Demonstração do teorema}

Podemos escrever: $Q=q\left(D^{\prime}\right)+2 \cos x_{1}=q\left(D^{\prime}\right)+e^{i x_{1}}+e^{-i x_{1}}$.

Seja $u \in \mathcal{D}^{\prime}\left(T^{n}\right)$ tal que $Q u=f \in C^{\infty}\left(T^{n}\right)$.

Substituindo as expansões em séries de Fourier de $u$ e $f$ na equação acima, teremos: $\forall k=\left(k_{1}, k^{\prime}\right) \in \mathbf{Z}^{n}$, onde $k^{\prime} \in \mathbf{Z}^{n-1}$, vale:

$$
q\left(k^{\prime}\right) \hat{u}(k)+\hat{u}\left(k-e_{1}\right)+\hat{u}\left(k+e_{1}\right)=\hat{f}(k),
$$

onde $e_{1}=(1,0, \ldots, 0) \in Z^{n}$.

Para cada $k^{\prime} \in \mathbf{Z}^{n-1}$ fixado, façamos:

$$
k_{1}=j ; j \in \mathbf{Z} ; \quad a_{j}=\frac{q\left(k^{\prime}\right)}{i}=c ; f_{j}=\hat{f}\left(j, k^{\prime}\right) ; v_{j}=i^{j} \hat{u}\left(j-1, k^{\prime}\right)
$$


e chegaremos à equação:

$$
v_{j+2}=c v_{j+1}+v_{j}-f_{j}, j=0, \pm 1, \pm 2, \ldots
$$

Definimos, para $j=1,2, \ldots, p_{j}$ e $q_{j}$ como no lema (3.1), os quais, neste caso, dependem de $k^{\prime}$, mas ainda verificam o resultado daquele lema:

$$
p_{j+1} q_{j}-p_{j} q_{j+1}=(-1)^{j}, j=1,2, \ldots, \forall k^{\prime} \in \mathbf{Z}^{n-1} .
$$

Por hipótese, temos: $\left|a_{j}\right|=|c|=\left|q\left(k^{\prime}\right)\right| \geq 2+\tau, \tau>0$, para todo $k^{\prime} \in \mathbf{Z}^{n-1}$. Logo, $\exists K>1$, independente de $j$ e $k^{\prime}$, tal que $\left|a_{j}\right| \geq 1+K$, para $j \geq 1$, e as hipóteses do lema (3.2) continuam válidas. Portanto, para todo $k^{\prime} \in \mathbf{Z}^{n-1}$ e todo $j \geq 4$, vale:

$$
\left|q_{j}\right| \geq K^{j-3} ;\left|p_{j}\right| \geq K^{j-3}
$$

Definimos

$$
t=\lim _{j \rightarrow \infty} \frac{p_{j}}{q_{j}}=\sum_{j=2}^{\infty} \frac{(-1)^{j}}{q_{j} q_{j+1}} .
$$

e pelo lema (3.3), existem $K_{0}>0$ e $j_{0} \geq 1$, independentes de $j$ e $k^{\prime}$, tais que:

$$
\left|p_{j}-t q_{j}\right| \leq K_{0}\left|q_{j}\right|^{-1}, \text { para } j \geq j_{0}
$$

A solução da equação $v_{j+2}=c v_{j+1}+v_{j}-f_{j}, j=1,2, \ldots, \operatorname{com} v_{1}=\alpha ; v_{2}=\beta$, segue como no lema (3.4), com a diferença de que os termos $t, p_{\nu}, q_{\nu}$ agora dependem de $k^{\prime}$.

Novamente, podemos escrever:

$$
v_{j}=\alpha p_{j}+\left(\beta-\gamma_{1}\right) q_{j}+r_{j}, \text { onde } r_{j}=T_{1}+T_{2}-f_{j-2}
$$

e, observando que as estimativas obtidas para $T_{1}$ e $T_{2}$ no teorema (3.1) só dependiam das desigualdades dos lemas (3.2) e (3.3), e estas continuam válidas, segue que $T_{1}, T_{2}$ decrescem rapidamente quando $j,\left|k^{\prime}\right| \rightarrow \infty$.

Como $u \in \mathcal{D}^{\prime}\left(T^{n}\right)$, da mesma maneira que na seção (3.3), concluímos que $\alpha t+\beta-\gamma_{1}=0$.

Resolvendo a equação de diferenças para $j \leq 0$, chegaremos à equivalente: $w_{j+2}=c w_{j+1}+w_{j}+(-1)^{j} f_{-j}$, com as condições iniciais: $w_{1}=-v_{1}=-\alpha \mathrm{e}$ $w_{2}=v_{0}=\beta+i q\left(k^{\prime}\right) \alpha+f_{0}$. 
Pelo lema (3.4), a solução desta última é dada por:

$$
w_{j}=-\alpha p_{j}+\left(\beta+f_{0}-i \alpha q\left(k^{\prime}\right)\right) q_{j}+\tilde{\varphi},
$$

onde $\tilde{\varphi_{1}}=\tilde{\varphi_{2}}=0$ e para $j \geq 3$ :

$$
\tilde{\varphi_{j}}=(-1)^{j} f_{2-j}-\sum_{\mu=1}^{j-3} f_{-\mu}\left\{p_{j} q_{\mu+1}-q_{j} p_{\mu+1}\right\}
$$

ou ainda,

$$
w_{j}=-\alpha p_{j}+\left(\beta+f_{0}+\gamma_{2}+i \alpha q\left(k^{\prime}\right)\right) q_{j}+\tilde{r_{j}},
$$

onde $\tilde{r}_{j}=\tilde{T}_{1}+\tilde{T}_{2}+(-1)^{j} f_{2-j}$ decai rapidamente quando $j,\left|k^{\prime}\right| \rightarrow \infty$.

Do fato de $u \in \mathcal{D}^{\prime}$, segue que:

$$
-\alpha t+\beta+f_{0}+\gamma_{2}+i \alpha q\left(k^{\prime}\right)=0
$$

e, junto com:

$$
\alpha t+\beta-\gamma_{1}=0
$$

temos:

$$
\alpha=\frac{\gamma_{1}+\gamma_{2}+f_{0}}{2 t-i q\left(k^{\prime}\right)} ; \beta=\frac{\left(t-i q\left(k^{\prime}\right)\right) \gamma_{1}-\gamma_{2} t-t f_{0}}{2 t-i q\left(k^{\prime}\right)}
$$

Observemos que, novamente, $\alpha$ e $\beta$ não dependem de $j$. Resta-nos mostrar que $\alpha$ decresce rapidamente quando $j,\left|k^{\prime}\right| \rightarrow \infty$.

Para isto, vejamos que $|t|<1$.

De fato, temos:

$$
|t|=\lim _{j \rightarrow \infty} \frac{\left|p_{j}\right|}{\left|q_{j}\right|} \text { e sabemos que: }
$$

\begin{tabular}{|l|l|}
\hline$q_{1}=0$ & $p_{1}=1 ; p_{2}=0$ \\
$q_{2}=1$ & $p_{3}=c p_{2}+p_{1}=1$ \\
$q_{3}=c q_{2}+q_{1}=c$ & $p_{4}=c p_{3}+p_{2}=c$ \\
$q_{4}=c q_{3}+q_{2}=c^{2}+1$ & $p_{5}=c p_{4}+p_{3}=c^{2}+1$ \\
$\vdots$ & $\vdots$ \\
\hline
\end{tabular}


Dai, concluímos que $q_{j}=p_{j+1}$, se $j \geq 2$. Logo:

$$
|t|=\lim _{j \rightarrow \infty} \frac{\left|p_{j}\right|}{\left|p_{j+1}\right|}
$$

e como $\left|p_{j+1}\right| \geq K\left|p_{j}\right|$, se $j \geq 3$, com $K>1$, temos:

$$
|t| \leq \frac{1}{K}<1
$$

Assim:

$$
\left|2 t-i q\left(k^{\prime}\right)\right| \geq|| q\left(k^{\prime}\right)|-| 2 t|| \geq|| q\left(k^{\prime}\right)|-2| \geq \tau
$$

e, portanto,

$$
|\alpha| \leq \frac{\left|\gamma_{1}+\gamma_{2}+f_{0}\right|}{\left|2 t-i q\left(k^{\prime}\right)\right|} \leq \frac{1}{\tau}\left|\gamma_{1}+\gamma_{2}+f_{0}\right|
$$

e podemos ver que $\gamma_{1}=\gamma_{1}\left(k^{\prime}\right) ; \gamma_{2}=\gamma_{2}\left(k^{\prime}\right)$ e $f_{0}=\hat{f}\left(0, k^{\prime}\right)$ decrescem rapidamente quando $\left|k^{\prime}\right| \rightarrow \infty$, da mesma maneira que na seção (3.3).

$\mathrm{E}$, com isso, podemos mostrar que $\hat{u}\left(j, k^{\prime}\right)$ tem decrescimento rápido quando $j,\left|k^{\prime}\right| \rightarrow \infty$ e concluímos que $u \in C^{\infty}\left(T^{n}\right)$.

A seguir, enunciaremos um outro resultado bastante significativo tratado em [Yos], o qual não será demonstrado neste trabalho:

\subsubsection{Outra classe de operadores}

Sejam $m$ e $s$ inteiros tais que $m \geq s \geq 1$. Consideremos o operador em $T^{n}$ :

$$
\text { (*) } \quad P u=\sum_{|\alpha| \leq m} a_{\alpha} D^{\alpha} u+\sum_{|\beta| \leq m-s} b_{\beta}(x) D^{\beta} u=f(x),
$$

onde $a_{\alpha} \in \mathrm{C}$ e $b_{\beta}, f(x) \in C^{\infty}\left(T^{n}\right)$.

Assumimos que

$$
\hat{b}_{\beta}(0)=(2 \pi)^{-n} \int_{T^{n}} b_{\beta}(x) d x=0, \forall \beta \operatorname{com}|\beta| \leq m-s .
$$

Tomemos as expansões em séries de Fourier:

$$
b_{\beta}(x)=\sum_{k \in \mathbb{Z}^{n}} \hat{b}_{\beta}(k) e^{i k . x}
$$


Definimos $\Gamma_{p}$ como sendo a envoltória convexa do fecho do conjunto:

$$
A=\left\{t k ; t \geq 0, \hat{b}_{\beta}(k) \not \equiv 0 \text { para algum } \beta:|\beta| \leq m-s\right\} \cup\{0\} .
$$

Seja $p(\xi)=\sum_{|\alpha| \leq m} a_{\alpha} \xi^{\alpha}$ e denotemos a $m$-ésima parte homogênea de $p(\xi)$ por $p_{m}(\xi)$, assumindo que $p_{m}(\xi) \not \equiv 0$.

Suponhamos que sejam válidas as condições:

- (B.1) $\Gamma_{p}$ é próprio, i.e., $\Gamma_{p}$ não contém nenhuma reta completa $\left(\Gamma_{p}\right.$ pode conter apenas semi-retas).

- (B.2) $p_{m}(\xi) \neq 0$ para todo $\xi \in \Gamma_{p} \backslash\{0\}$ (ou seja, exige-se aqui certa "eliticidade" do operador em $\Gamma_{p}$ ).

- (B.3) A multiplicidade das raízes da equação $p_{m}(\xi)=0$ não excede $s$ dado em $\left(^{*}\right)$.

Então vale o seguinte teorema:

Teorema 3.3 Se valem (B.1), (B.2) e (B.3), então $P$ é (GH) se, e somente se, existem constantes $c \in \mathbf{R}, K_{1}>0$ e $K_{2}>0$ tais que :

(B.4) $|p(k)|>K_{1}(1+|k|)^{c}, \quad \forall k \in \mathbf{Z}^{n},|k| \geq K_{2}$.

Observemos que (B.4) é análoga à condição (LM) do primeiro teorema do capítulo 2, mesmo aqui se tratando de um operador mais geral (no sentido de que pode apresentar muitos coeficientes variáveis).

A prova da suficiência envolve a construção de operadores pseudo-diferenciais que forneçam estimativas das normas de Sobolev de uma solução $u \in \mathcal{D}^{\prime}$ da equação $P u=f, \operatorname{com} f \in C^{\infty}\left(T^{n}\right)$. A obtenção de tais estimativas exige um trabalho árduo.

A prova da necessidade é feita por contrapositiva e segue a mesma idéia, utilizando-se de outras estimativas e de um resultado que envolve idéias bastante geométricas (lema (3.1) de [Yos]). 


\section{Apêndice A}

Resultado: As topologias descritas, respectivamente, pelas semi-normas de Sobolev:

$$
\|\varphi\|_{1, r}=\left[\sum_{k \in \mathbb{Z}^{n}}(1+|k|)^{2 r}|\hat{\varphi}(k)|^{2}\right]^{1 / 2}
$$

e

$$
\|\varphi\|_{2, \tau}=\left[\sum_{k \in \mathbb{Z}^{n}}|k|^{2 \tau}|\hat{\varphi}(k)|^{2}\right]^{1 / 2}
$$

coincidem em $C_{0}^{\infty}\left(T^{n}\right)$.

\section{Prova:}

i) Inicialmente, observemos que uma sequência $\left(f_{n}\right)_{n \in \mathbb{N}} \in C^{\infty}\left(T^{n}\right)$ converge para zero neste espaço se, e somente se, ela converge para zero em cada uma das semi-normas que descrevem sua topologia.

De fato:

Vimos que $C^{\infty}\left(T^{n}\right)$ é um espaço de Fréchet cuja métrica pode ser dada por:

$$
d(f, g)=\sum_{j=0}^{\infty} \frac{1}{2^{j}} \frac{p_{j}(f-g)}{1+p_{j}(f-g)},
$$

onde $p_{j}(f)=\|f\|_{2, j}, j=0,1,2, \ldots$ (utilizamos aqui, o fato de $C^{\infty}\left(T^{n}\right)$ ser isomorfo e homeomorfo a $S\left(\mathbf{Z}^{n}\right)$, através do operador $\wedge$ ).

Assim, suponhamos que $f_{n} \rightarrow 0$ em $C^{\infty}\left(T^{n}\right)$. Então, dado $\varepsilon, 0<\varepsilon<1 / 2$, existe $n_{0} \in \mathbf{N}$ tal que:

$$
\begin{array}{r}
d\left(f_{n}, 0\right)=\sum_{j=0}^{\infty} \frac{1}{2^{j}} \frac{p_{j}\left(f_{n}\right)}{1+p_{j}\left(f_{n}\right)}<\varepsilon, \forall n \geq n_{0} \Rightarrow \\
\forall j \geq 0, \frac{p_{j}\left(f_{n}\right)}{1+p_{j}\left(f_{n}\right)}<\varepsilon \Leftrightarrow p_{j}\left(f_{n}\right)<\varepsilon+\varepsilon p_{j}\left(f_{n}\right) .
\end{array}
$$




$$
\Leftrightarrow 0 \leq p_{j}\left(f_{n}\right)<\frac{\varepsilon}{1-\varepsilon}<2 \varepsilon, \forall n \geq n_{0}
$$

donde concluimos que: $\forall j \geq 0, \lim _{n \rightarrow \infty} p_{j}\left(f_{n}\right)=0$.

Reciprocamente, se $p_{j}\left(f_{n}\right) \rightarrow 0, \forall j=1,2, \ldots$, então:

$$
\text { dado } \varepsilon>0, \exists n_{0}=n_{0}(j) \text { tal que } p_{j}\left(f_{n}\right)<\varepsilon / 4, \forall n \geq n_{0} .
$$

Como

$$
\begin{gathered}
\sum_{j=0}^{\infty} \frac{1}{2^{j}} \frac{p_{j}\left(f_{n}\right)}{1+p_{j}\left(f_{n}\right)}<\infty, \text { existe } j_{0}>0 \text { tal que : } \\
\sum_{j=j_{0}}^{\infty} \frac{1}{2^{j}} \frac{p_{j}\left(f_{n}\right)}{1+p_{j}\left(f_{n}\right)}<\frac{\varepsilon}{2} .
\end{gathered}
$$

Tomando $n_{1}=\max \left\{n_{0}(0), \ldots, n_{0}\left(j_{0}-1\right)\right\}$, teremos:

para todo $n \geq n_{1}$ vale:

$$
\begin{aligned}
\sum_{j=0}^{\infty} \frac{1}{2^{j}} \frac{p_{j}\left(f_{n}\right)}{1+p_{j}\left(f_{n}\right)} & =\sum_{j=0}^{j_{0}-1} \frac{1}{2^{j}} \frac{p_{j}\left(f_{n}\right)}{1+p_{j}\left(f_{n}\right)}+\sum_{j=j_{0}}^{\infty} \frac{1}{2^{j}} \frac{p_{j}\left(f_{n}\right)}{1+p_{j}\left(f_{n}\right)} \\
& \leq \sum_{j=0}^{j_{0}-1} \frac{p_{j}\left(f_{n}\right)}{2^{j}}+\frac{\varepsilon}{2}<\frac{\varepsilon}{4} \sum_{j=0}^{j_{0}-1} \frac{1}{2^{j}}+\frac{\varepsilon}{2} \\
& \leq \frac{\varepsilon}{2}+\frac{\varepsilon}{2}=\varepsilon .
\end{aligned}
$$

ii) Como $C_{0}^{\infty}\left(T^{n}\right)$ tem a topologia induzida de $C^{\infty}\left(T^{n}\right)$, então uma sequência $\left(f_{n}\right)_{n \in \mathrm{N}}$ convergirá em $C_{0}^{\infty}\left(T^{n}\right)$ se, e somente se, ocorrer a convergência em cada uma das semi-normas $p_{j}$.

Assim, mostraremos que as semi-normas de Sobolev $\|\cdot\|_{1, j}$ e $\|\cdot\|_{2, j}$ são equivalentes em $C_{0}^{\infty}\left(T^{n}\right)$, para cada $j=0,1,2, \ldots$ e, então, concluímos a prova.

Com efeito, se $f \in C_{0}^{\infty}\left(T^{n}\right)$, então $\hat{f}(0)=0$.

Logo:

$$
\sum_{k \in \mathbb{Z}^{n}}(1+|k|)^{2 j}|\hat{f}(k)|^{2}=\sum_{k \in \mathbb{Z}^{n} \backslash\{0\}}(1+|k|)^{2 j}|\hat{f}(k)|^{2}=\|f\|_{1, j}
$$


Assim,

$\forall j \geq 0,\|f\|_{2, j}=\left[\sum_{k \in Z^{n}}|k|^{2 j}|\hat{f}(k)|^{2}\right]^{1 / 2} \leq\left[\sum_{k \in Z^{n}}(1+|k|)^{2 j}|\hat{f}(k)|^{2}\right]^{1 / 2}=\|f\|_{1, j}$

e ainda, $\forall j \geq 0$, para todo $k \in \mathbf{Z}^{n} \backslash\{0\}$, vale:

$(1+|k|)^{2 j}|\hat{f}(k)|^{2} \leq(2|k|)^{2 j}|\hat{f}(k)|^{2}$, donde temos:

$$
\begin{aligned}
\|f\|_{1, j}=\left[\sum_{k \in \mathbb{Z}^{n} \backslash\{0\}}(1+|k|)^{2 j}|\hat{f}(k)|^{2}\right]^{1 / 2} & \leq 2^{j}\left[\sum_{k \in \mathbb{Z}^{n} \backslash\{0\}}|k|^{2 j}|\hat{f}(k)|^{2}\right]^{1 / 2} \\
& =2^{j}\|f\|_{2, j}
\end{aligned}
$$

e, portanto,

$\|f\|_{2, j} \leq\|f\|_{1, j} \leq 2^{j}\|f\|_{2, j}, \quad$ para todo $j=0,1,2, \ldots$ 


\section{Bibliografia}

[Berg] Bergamasco, A.P., Hipoeliticidade Global para Algumas Classes de Operadores, dissertação de Livre-Docência, ICMSC-USP, 1989.

[G-W] Greenfield, S.J. and Wallach, N.R., Global Hypoellipticity and Liouville Numbers, Proceedings Am. Math. Soc., 31, 112-114, 1972.

[Hale] Hale, J., Ordinary Differential Equations, Wiley Interscience, 91$92,1969$.

[Hou] Hounie, J., Teoria Elementar das Distribuições, 12o Colóquio Brasileiro de Matemática, IMPA, 1979.

[Ior] Iório Jr., R. e lório, V.M., Equações Diferenciais Parciais: uma introdução, IMPA - Projeto Euclides, 1988.

[Mos] Moser, J., Quasi-periodic Solutions of Nonlinear Elliptic Partial Differential Equations, Boletim da Sociedade Brasileira de Matemática, Vol.20, no 1, 29-45, 1989.

[Rud] Rudin, W., Functional Analysis, McGraw-Hill Book Company, 4849, 1973.

[Yos] Yoshino, M., A Class of Globally Hypoelliptic Operators on the Torus, Math. Z., 201, 1-11, 1989.

[War] Warner, F.W., Foundations of Differentiable Manifolds and Lie Groups, Springer, 231-238, 1971.

[Zan] Zani, S.L., Hipoeliticidade Global para Operadores de Segunda Ordem, dissertação de mestrado, ICMSC-USP, 1988. 